\title{
Circular RNA screening identifies circMYLK4 as a regulator of fast/slow myofibers in porcine skeletal muscles
}

\section{Haigang Cao}

North West Agriculture and Forestry University

Jieming Liu

North West Agriculture and Forestry University

Tianning Du

North West Agriculture and Forestry University

Yihao Liu

North West Agriculture and Forestry University

Xiaoyu Zhang

North West Agriculture and Forestry University

\section{Yuan Guo}

North West Agriculture and Forestry University

Jie Wang

North West Agriculture and Forestry University

Xiaomin Zhou

North West Agriculture and Forestry University

Xiao Li

North West Agriculture and Forestry University

\section{Gongshe Yang}

North West Agriculture and Forestry University

Xine Shi ( $\nabla$ xineshi@nwafu.edu.cn )

North West Agriculture and Forestry University

\section{Research Article}

Keywords: high throughput sequencing, pig, meat quality, muscle fiber type, circMYLK4

Posted Date: December 17th, 2020

DOI: https://doi.org/10.21203/rs.3.rs-120895/v1 
License: (c) (i) This work is licensed under a Creative Commons Attribution 4.0 International License. Read Full License

Version of Record: A version of this preprint was published at Molecular Genetics and Genomics on November 16th, 2021. See the published version at https://doi.org/10.1007/s00438-021-01835-5. 


\section{Abstract}

Background: The myofiber type is related to the quality of meat; specifically, slow-oxidized myofiber helps to increase the tenderness and juiciness of meat. An increasing number of studies have shown that circRNAs play a key role in skeletal muscle development. However, the key circRNAs that regulate myofiber types and their roles are still poorly understood.

Results: A total of 40757 circRNAs were identified from the longissimus dorsi (LD) and the soleus (Sol) muscles, of which 10388 were co-expressed in the two muscles. Further analysis found 181 differentially expressed circRNAs in the LD compared with Sol. Functional enrichment analysis showed that target genes of differentially expressed circRNA-sponge miRNAs were enriched in the AMPK, FoxO and PI3K-Akt signaling pathways. In addition, we focused on a novel circRNA-circMYLK4. CircMYLK4 significantly increased the mRNA and protein levels of slow muscle marker genes and caused the flesh to turn red.

Conclusion: Our study laid an essential foundation for further research on circRNAs in myofiber type conversion and the achievement of higher meat quality.

\section{Background}

Meat quality has been a major focus over the last decades because of both increasing living standards and population growth. As one of the dietary structures in our daily life, the quality of pork affects everyone's health. Therefore, improving quality without decreasing meat production is a promising research direction for porcine breeding scientists. Pork quality is influenced by multiple factors, and a growing number of studies have shown that muscle fiber types are closely related to quality [1]. Compared to fast muscle fiber, slow muscle fiber improves meat quality by affecting meat $\mathrm{pH}[2,3,4]$, meat color $[5,6,7]$, and water-holding capacity $[3,8]$. Hence, incorporating slow muscle fiber is a powerful strategy to improve meat quality.

It is generally believed that the total number of muscle fibers remains basically unchanged after the birth of an animal but that the muscle fiber type transform during the growth process. Transformation is the result of multiple factors, such as internal regulatory factors, which are mainly associated with signaling pathways and related cytokines, among which circular RNAs (circRNAs) play an important role. CircRNAs are a type of covalently closed circular RNA molecules formed by reverse splicing of the precursor mRNA without 5' cap and 3' poly (A) tail structures [9]. Previous studies have shown that circRNAs are present in tissues and cells of various biological organisms. For example, circRNAs are abundantly expressed in skeletal muscles of monkey and directly or indirectly regulate important factors of muscle [10]. Moreover, circLMO7 regulates the proliferation and differentiation of myoblasts by sponging miR-378a-3p [11]. Additionally, the protein translated by circZNF609 is involved in the growth and development of muscle [12]. More importantly, other studies have shown that circRNAs mainly regulate skeletal muscle growth development and muscle fiber type conversion during 0-30 d after birth and regulate skeletal muscle glucose metabolism and calcium ion signal during 30-240 d, but their regulatory mechanism is still 
unclear [13]. It is easy to conclude that circRNAs play an important role in skeletal muscle development and muscle fiber type switching according to previous literature; however, the roles and regulatory mechanisms of many circRNAs remain unknown.

In this study, a high-throughput sequencing technique was used to construct a cDNA library of skeletal muscle, including fast muscle (longissimus dorsi, LD) and slow muscle (soleus, Sol). After quality control filtration of the data, 181 differentially expressed circRNAs were identified by screening. Interestingly, we were attracted to a special circRNA, circMYLK4, which was highly expressed in slow muscle. Thus, we investigated the effects of circMYLK4 on muscle fiber types in pigs by injecting adeno-associated virus (AAV). As a result, circMYLK4 promoted the development of slow muscle fibers. These results provide a useful resource for further exploration of circRNA in transformation of muscle fiber types and improvement of meat quality.

\section{Methods}

\section{Ethics statement}

All animal care, slaughter and sample collection procedures was carried out in strict accordance with the protocol approved by the Institutional Animal Care and Institutional Ethic Committee of Northwest A\&F University (NWAFU-314020038).

\section{RNA extraction and RNase R treatment}

The longissimus dorsi and soleus muscle samples were taken from three 180-day-old healthy large white pigs of the same parity and body weight. Total RNA was extracted from muscle samples by the TRIzol reagent according to the manufacturer's instructions (Takara, Kyoto, Japan). The quality and concentration of RNA were detected by a NanoDrop 2000 instrument (Thermo Scientific, Waltham, MA, USA). Subsequently, ribosomal RNA (rRNA) was removed by Epicenter Ribo-zero rRNA Removal Kit (Epicenter, USA). Then, the residue RNAs were treated with RNase R (Epicenter, USA) to degrade the linear RNAs and were purified using RNeasy MinElute Cleanup Kit (Qiagen, Germany).

\section{Library construction and sequencing}

Ribosome RNA and linear RNA were removed to retain circRNAs. We used fragmentation buffer (Ambion, Foster City, CA, USA) to fragment the enriched circRNA and reverse transcribed the circRNA into cDNA using random primers. The second strand of CDNA was synthesized by DNA polymerase I, RNase $\mathrm{H}$, dNTPs and buffer. The double-stranded cDNA was purified with the QiaQuick PCR extraction kit (Qiagen, USA), end repaired, polyadenylated, and ligated to Illumina sequencing adapters. Then, we used Uracil-NGlycosylase (UNG) to digest the second-strand cDNA. The fragments were purified by VAHTSTM DNA Clean Beads and enriched by PCR amplification. Finally, the library products were sequenced via Illumina HiSeqTM2500. 
The original image file (BCL) obtained by sequencing was base-recognized and converted into raw data of FASTQ format. Then, clean data was obtained by filtering raw data, de-joining sequences, treating lowquality reads, quality assessing, and removing ribosome RNA. The clean data were compared with the reference genome twice. First, a normal alignment was performed by Tophat2 software, and the fusion gene was not included for comparison so that the reads that could not be directly compared to the genome were obtained. Then the Tophat-fusion module of Tophat2 software was used to perform fusion gene alignment on unmapped reads, to obtain potential circular reads. The Tophat-fusion results were filtered according to circRNA reads with reverse splicing points and sequences of splicing sites that are usually $G T / A G$, to identify circRNA more accurately $[14,15,16]$.

\section{Expression profiling and analysis of differentially expressed circRNAs}

We used HTseq software to calculate the count value for each circRNA to calculate its reads per million mapped reads (RPM) value, and count the number of splicing site sequences. DESeq was used in subsequent analysis to calculate the p-value and q-value of the gene in the comparison group, and the differentially expressed circRNAs between the samples were selected by the difference multiplier (log2 (Fold change) $>1$ ) and the significance level (q-value $<0.05$ ).

\section{Network construction and KEGG enrichment analysis}

We used miRanda, RNAhybrid, and TargetScan software to predict miRNA binding to exonic circRNA, and then we used Cytoscape software to construct a circRNA-miRNA interaction network. Additionally, miRanda, pita, and RNAhybrid software was used to predict the target genes of these miRNAs, and then biological pathway enrichment analysis was conducted on the target gene sets based on the Kyoto Encyclopedia of Genes and Genomes (KEGG) biological pathway database.

\section{In vivo injection of circMYLK4-AAV}

Construction of the circMYLK4 overexpression vector and virus packaging were completed by Guangzhou geneseed. The circMYLK4-AAV virus titer packaged by geneseed is $1 \times 10^{13} \mathrm{GC} / \mathrm{mL}$. We selected five 12day-old piglets for gastrocnemius injection. Each piglet was injected with $50 \mu \mathrm{L}$ of virus titer of $1 \times 10^{13}$ $\mathrm{GC} / \mathrm{mL}$ in the left leg (diluted $50 \mu \mathrm{L}$ of virus into $2 \mathrm{~mL}$ for easy injection), and the same amount of control virus was injected in the right leg. The piglets were sacrificed four weeks after injection, and the gastrocnemius, semitendinosus, semimembranosus and soleus muscles were separately collected for subsequent experiments.

\section{Real-time quantitative polymerase chain reaction (RT-qPCR) and Western blot}

RT-qPCR was performed on an Applied Biosystems real-time quantitative PCR machine using SYBR green master mix (Vazyme, China) according to the manufacturer's protocol. Protein extraction and Western blot analysis of tissues were performed according to our previous methods [17]. Antibodies against 
MyHC $₫$ were from Developmental Studies Hybridoma Bank (DSHB, USA). Antibodies against $\beta$-tubulin were from Boster.

\section{Statistical analysis}

The experimental data are expressed as the mean \pm SEM. All experimental data were analyzed by ANOVA and significance test using GraphPad Prism 7. ${ }^{\star} P<0.05,{ }^{* \star} P<0.01$.

\section{Results}

\section{Overview of circRNA profiles in LD and Sol skeletal muscle}

To determine the identity, abundance and differential expression of circRNAs in different types of skeletal fibers, we profiled circRNAs in slow-type-enriched Sol and fast-type-enriched LD of large white pigs. Ribodepleted total RNA-Seq libraries were prepared and sequenced according to the flow chart (Fig. 1A). We identified a considerable number of RNAs in LD and Sol muscles when considering total RNA libraries (Table 1). A total of 40757 candidate circRNAs were identified by at least one unique back-spliced read. We found that 15627 and 14742 circRNAs were specifically expressed in LD and Sol muscles respectively, and 10388 were expressed in both muscles (Fig. 1B). For both the LD and Sol samples, the circRNAs are mostly comprised of exonic, intronic and intergenic sequences, as determined based on their mapping to the genome, whereas a smaller fraction of circRNAs also contains upstream, downstream and UTRs (Fig. 1C).

Table 1 Summary of reads mapping to the reference genome and identification of circular RNAs.

\begin{tabular}{ccc}
\hline Samples & LD & Sol \\
\hline Raw reads & $99,987,084$ & $82,541,576$ \\
Clean reads & $86,645,908$ & $72,841,678$ \\
Mapped reads & $73,928,442(85.52 \%)$ & $63,016,935(86.77 \%)$ \\
\hline Mapped reads(unfusion) & $62,626,818(72.45 \%)$ & $53,855,038(74.15 \%)$ \\
\hline Candidate back-spliced junction reads & $1,000,194(1.154 \%)$ & $880,632(1.209 \%)$ \\
\hline Realign post reads & $424,828(0.490 \%)$ & $328,353(0.451 \%)$ \\
\hline Circular RNA number & 26,015 & 25,130 \\
\hline Circular RNA number(reads>1) & 20,351 & 19,483 \\
\hline
\end{tabular}

\section{Identification and characteristics of circRNAs expressed in LD and Sol}

To further estimate the characteristics of circRNAs, we analyzed the circRNA sequences. Standard metrics to characterize the length of circRNAs detected in this study (minimum, maximum, mean, median, and total length) are provided in Table 2. Most circRNAs were no more than 1000 nucleotides (nt), and the 
median length was $439 \mathrm{nt}$ (Fig. 2A). According to their host gene location, the 40757 circRNAs were widely distributed across chromosomes except for the $Y$ chromosome, and the number of circRNAs increased with chromosome length (Fig. 2B). According to the histogram depicting flanking intron lengths, the length of flanking intron regions of most circRNAs was approximately $10^{3}-10^{4} \mathrm{nt}$, indicating that long flanking introns are necessary for circRNA formation (Fig. 2C-D). A total of 21193 (52.0\%) circRNAs were composed of one to eight exons, among which 4606 (11.3\%) contained one exon. Furthermore, 12204 (29.9\%) circRNAs contained more than fifteen exons (Fig. 2E). We identified 40757 circRNAs generated from 8009 host genes, indicating that most genes can produce multiple circRNAs. There were 1007 (12.6\%) circRNA-producing genes that generated a single circRNA, whereas 26.6\% produced more than fifteen (Fig. 2F).

Table 2 Results from the assembly of circRNAs.

Item circRNA Min.length Mean length Median length Max. length Total length

\begin{tabular}{lllllll}
\hline Number & 40757 & 34 & 2734 & 439 & 99934 & 111433971 \\
\hline
\end{tabular}

\section{Analysis of differentially expressed circRNA between LD and Sol}

Based on the analysis of circRNA expression, we found 181 significantly differentially expressed circRNAs when comparing the libraries derived from the LD and Sol (Additional File). To further explore the potential functions of circRNA, we constructed a clustered heatmap (Fig. 3A). Although several circRNAs showed the same expression pattern, most of them were differentially expressed between the LD and Sol. Based on the expression levels of circRNAs in paired samples (LD to Sol ratio), 90 circRNAs were downregulated, and 91 circRNAs were upregulated in the LD (Fig. 3B).

\section{Construction of circRNA-miRNA interaction network}

A previous study has shown that circRNAs composed of only exons can play a role in the cytoplasm by sponging microRNA (miRNA). We randomly selected 11 differentially expressed exonic circRNAs and predicted the miRNAs they might sponge by miRanda, RNAhybrid and TargetScan. Then, we constructed an interactive network between these 11 circRNAs and the sponged miRNAs (Fig. 4). The whole interaction network included 11 circRNAs, 111 miRNAs and 146 relationship lines.

\section{KEGG pathway enrichment analysis}

To further analyze the regulatory mechanisms of differentially expressed circRNAs in different types of skeletal muscle fibers, we predicted the sponge miRNAs of the differentially expressed circRNAs and analyzed the target genes of miRNAs. Then, we performed pathway enrichment analysis of the predicted target genes (Fig. 5). A total of 30 signaling pathways were enriched for these genes, among which metabolic pathways were the most abundant pathway. Markedly, some pathways were closely related to 
muscle fiber development, resulting in the transformation of muscle fiber types, such as the AMPK signaling pathway, FoxO signaling pathway, and PI3K-Akt signaling pathway.

\section{Validation of differentially expressed circRNAs by RT-qPCR}

To validate the data reliability of differentially expressed circRNAs detected by sequencing, we randomly selected 9 differentially expressed circRNAs and amplified their junction regions using specific RT-qPCR primers. RT-qPCR revealed two upregulated and seven downregulated circRNAs, as shown in Fig. 6, which clearly showed that expression patterns of the nine selected circRNAs were consistent with the results of RNA-Seq. These results demonstrated the accuracy and reliability of high-throughput sequencing.

\section{Overexpression of circMYLK4 promotes the development of slow muscle fibers}

We analyzed novel circRNAs in many aspects and selected circMYLK4, which was named after its host gene MYLK4 located on chromosome 7, for its high expression in Sol for further analysis. To investigate whether this circRNA could be a regulatory factor during muscle fiber development, we injected piglets with circMYLK4-AAV. Then, we collected gastrocnemius (GAS), semimembranosus (SB), semitendinosus (SM) and soleus (Sol) samples for analysis 4 weeks after injection. The expression level of circMYLK4 in AAV group was significantly higher than that in control group (Fig. 7A). The results of the meat color assessment showed that circMYLK4 reduced the brightness of meat and improved the redness and yellowness of meat (Fig. 7B). In support of this phenomenon, circMYLK4 overexpression significantly increased expression levels of the slow muscle marker gene $\mathrm{MyHCl}$, which was detectible at both the mRNA and protein levels (Fig. 7C-D). In addition, we also measured the mRNA of fiber type-related genes (Tnnt1, Tnni1, and Tnnc1), mitochondrial biogenesis genes (PGC-1 $a$ and Tfam), and mitochondrial respiratory chain genes (Atp5o, Cycs, and Ndufa5) by RT-qPCR. As expected, circMYLK4 overexpression increased the expression of the above genes (Fig. 7E-H). Collectively, these results established that circMYLK4 promoted the development of slow muscle fibers by regulating the expression of mitochondrial-related factors.

\section{Discussion}

The characteristics of skeletal muscle fiber are associated with the quality of meat [18]. Generally, skeletal muscle fibers can be divided into fast muscle fibers and slow muscle fibers according to their morphological and functional characteristics [19]. Slow muscle fibers are characterized by high content of mitochondria, lipids and myoglobin and a small diameter, while fast muscle fibers have the opposite characteristics [20]. Almost all skeletal muscles are composed of slow muscle fibers and fast muscle fibers. Studies have shown that a higher content of slow muscle fibers makes the meat ruddy, fresh and juicy, which improves the flavor of meat, while a higher content of fast muscle fibers makes the meat white and leads to reduced quality [18]. Therefore, increasing the ratio of slow muscle fiber can improve meat quality. CircRNAs were originally thought to be a by-product of splicing [21], but with the rapid development of high-throughput sequencing technology, their true nature is gradually becoming well known. In recent years, the research on circRNAs has mainly focused on human diseases in medicine, 
and there have been studies on skeletal muscle fibers in agricultural animals. Therefore, the in-depth exploration of circRNAs involved in muscle fiber development is a new direction for meat quality improvement research.

In this study, a total of 40757 circRNA were identified from the LD and Sol muscles, of which 10388 were co-expressed in the two muscles. Further, 181 differentially expressed circRNAs in the LD and Sol were identified. CircRNA may partially participate in the regulation of porcine skeletal muscle fiber types through signaling pathways such as AMPK, FoxO, and PI3K-Akt. More important, a novel circRNA, circMYLK4, could promote the development of slow muscle fibers. Overall, our findings provide scientific evidence that circRNAs regulate muscle fiber types.

In recent years, studies on circRNA in pigs have mainly focused on the sequencing identification stage. Huang studied the circRNA expression profiles of 70-day-old Jinhua pigs and Landrace pigs and identified 84864 circRNAs, of which 366 were differentially expressed and mainly enriched in lipid metabolism pathways [22]. This provides basic data for further study of the biological function of porcine liver circRNA. Liang performed a circRNA genome-wide analysis of nine organs (muscle, fat, liver, heart, spleen, lung, kidney, ovary, and testis) and skeletal muscles of three different development stages in Guizhou mini-pigs. A total of 5934 circRNAs were identified, of which 149 were related to muscle development, and the first porcine circRNAs database was constructed [13]. Sun performed highthroughput sequencing of longissimus miRNA, IncRNA, and circRNA of Landrace and Lantang pigs, and the results showed that 236 differentially expressed circRNAs were screened, among which 40 participated in sponge modulators of 26 miRNA-mediated ceRNA interactions [23]. Their study revealed a new post-transcriptional regulatory layer that could promote the understanding of the molecular mechanisms of muscle fiber development in different breeds of pigs. Shen studied the circRNA expression profiles of the psoas major muscle and the longissimus dorsi muscle of 178-day-old Qingyu pigs and identified 810 circRNAs, of which 137 were differentially expressed [24]. These circRNAs may be involved in the regulatory network of muscle fiber type. In our study, a total of 40757 circRNAs were identified, of which 181 were significantly differentially expressed. Overall, our identification results enriched the pig circRNA database, providing a basic reference for studying pig muscle fiber type transformation and improving meat quality.

In the characterization of circRNAs, we found that the length of circRNAs is mainly distributed approximately $400 \mathrm{bp}$, and the length of circRNAs is positively correlated with the corresponding chromosome length. This finding is similar to previous observations of bovine skeletal muscle samples [11]. This finding potentially indicates that the length and number of circRNAs are common features of skeletal muscle tissue. Additionally, we found that the length of the upstream and downstream flanking introns of circRNAs is mostly approximately $10000 \mathrm{bp}$. This is caused by the main loop-forming mechanism of circRNA, in which the flanking intron region contains repeats or complementary sequences, such as SINE [25] or ALU sequences [14, 26, 27], which requires the flanking introns to have a sufficiently long length to facilitate this event. Our results indicate that one parental gene can produce multiple circRNA isoforms through alternative splicing [14]. 
Skeletal muscle fiber types are regulated by a number of signaling pathways. Transformation of skeletal muscle fiber types is accompanied by changes in mitochondria and metabolism. Studies have shown that succinate induces skeletal muscle fiber remodeling by promoting mitochondrial biogenesis and aerobic oxidation [28]. Additionally, ferulic acid promotes the formation of slow muscle fibers by activating Sirt1 and AMPK to upregulate the expression of PGC-1 a [29]. 6-Gingerol stimulates AMPK/PGC-1a signaling pathway and accelerates a fast-to-slow-fiber type transition and muscle oxidative metabolism by increasing plasma adiponectin and muscle AdipoR1 [30]. Kitamura found that Foxo1 ablation in skeletal muscle results in increased formation of MyoD-containing (fast-twitch) muscle fibers and altered fiber type distribution at the expense of myogenin-containing (slow-twitch) fibers [31]. In our study, we found that target genes of differentially expressed circRNA-sponge miRNAs were enriched in the AMPK, FoxO and PI3K-Akt signaling pathways, potentially suggesting that these circRNAs regulate muscle fiber types through the indicated signaling pathways. Although there is no clear evidence that PI3K-Akt plays a role in muscle fiber types, some studies have shown that the PI3K-Akt signaling pathway is related to skeletal muscle development and atrophy [32,33].

Studies have shown that circMYLK produced by human myosin light chain kinase (MYLK) can competitively bind to miR-29a, abolishing miR-29a inhibition of VEGFA/VEGFR2 and downstream Ras/ERK signaling pathways, thereby promoteing epithelial-mesenchymal transition and bladder cancer development [34]. In this sequencing analysis, we found that circMYLK4 produced by myosin light chain kinase family member 4 (MYLK4) in pigs was significantly differentially expressed in different types of skeletal muscle fiber in pigs. Interestingly, circMYLK4 has a completely different sequence from circMYLK and is considered a new circRNA. By injecting circMYLK4-AAV into piglets, we found that circMYLK4 promotes the expression of slow muscle fiber marker genes and mitochondria-related genes. Our results require further study of the mechanism by which circMYLK4 upregulates slow muscle fibers.

\section{Conclusions}

In conclusion, we found 181 differentially expressed circRNAs between the longissimus dorsi and soleus muscles of pigs and identified a novel circRNA, circMYLK4, that promoted the formation of oxidized fibers (Fig. 8). These findings provide a theoretical basis for further study of myofiber types conversion and higher meat quality.

\section{Abbreviations}

CircRNA: Circular RNA

LD: Longissimus dorsi

Sol: Soleus

GAS: Gastrocnemius 
SB: Semimembranosus

SM: Semitendinosus

AAV: Adeno-associated virus

AMPK: Adenosine 5'-monophosphate (AMP)-activated protein kinase

FoxO: Forkhead box 0

PI3K-Akt: Phosphatidylinositol 3 kinase-protein kinase B

MyHC I: Myosin heavy chain I

Tnnt1: Troponin T1

Tnni1: Troponin I1

Tnnc1: Troponin C1

PGC-1a: Peroxisome proliferator-activated receptor gamma coactivator 1

Tfam: Transcription Factor A Mitochondrial

Atp5o: ATP Synthase Peripheral Stalk Subunit OSCP

Cycs: Cytochrome C Somatic

Ndufa5: NADH-Ubiquinone Oxidoreductase Subunit A5

MYLK4: Myosin light chain kinase family member 4

\section{Declarations}

\section{Acknowledgements}

We would like to thank Dr. Bo Xia and Dr. Weike Shaoyong for their technical assistance with this project.

\section{Funding}

This work was supported by grants from the National Natural Science Foundation (31772570).

\section{Availability of data and materials}

The datasets used and analysed during the current study available from the corresponding author on reasonable request. 
Author information

\section{Affiliations}

Key Laboratory of Animal Genetics, Breeding and Reproduction of Shaanxi Province $\square$ College of Animal Science and Technology, Northwest A\&F University, Yangling, Shaanxi 712100, China

Haigang Cao, Jieming Liu, Tianning Du, Yihao Liu, Xiaoyu Zhang, Yuan Guo, Jie Wang, Xiaomin Zhou, Xiao Li, Gongshe Yang, and Xin'e Shi

\section{Contributions}

HGC, JML and TND performed the experiments. HGC, YHL and XYZ analyzed the data. HGC, YG, JW and XMZ participated in the collection of samples. HGC, XL and XES wrote the manuscript. GSY and XES conceived and designed the experiments. All authors read and approved the final manuscript.

\section{Corresponding author}

Correspondence to Xin'e Shi.

\section{Ethics declarations}

\section{Ethics approval and consent to participate}

All animal care, slaughter and sample collection procedures was carried out in strict accordance with the protocol approved by the Institutional Animal Care and Institutional Ethic Committee of Northwest A\&F University (NWAFU-314020038).

\section{Consent for publication}

Not applicable.

\section{Competing interests}

The authors declare that they have no competing interests.

\section{References}

1. Lefaucheur L. A second look into fibre typing-relation to meat quality. Meat Science. 2010;84(2):257270.

2. Choi YM, Ryu YC, Kim BC. Influence of myosin heavy- and light chain isoforms on early postmortem glycolytic rate and pork quality. Meat Science. 2007;76(2):281-288.

3. Kim GD, Jeong JY, Jung EY, Yang HS, Lim HT, Joo ST. The influence of fiber size distribution of type IIB on carcass traits and meat quality in pigs. Meat Science. 2013;94(2):267-273. 
4. Ryu YC, Kim BC. Comparison of histochemical characteristics in various pork groups categorized by postmortem metabolic rate and pork quality. Journal of Animal Science. 2006;84(4):894-901.

5. Kauffman RG, van Laack RL, Russell RL, Pospiech E, Cornelius CA, Suckow CE, et al. Can pale, soft, exudative pork be prevented by postmortem sodium bicarbonate injection? Journal of Animal Science. 1998;76(12):3010-3015.

6. Kim G-D, Jeong J-Y, Hur S-J, Yang H-S, Jeon J-T, Joo S-T. The Relationship between Meat Color (CIE $L^{*}$ and $\left.a^{*}\right)$, Myoglobin Content, and Their Influence on Muscle Fiber Characteristics and Pork Quality. Korean Journal for Food Science of Animal Resources. 2010;30(4):626-633.

7. Renerre M. Review: Factors involved in the discoloration of beef meat. International Journal of Food Science \& Technology. 1990;25:613-630.

8. Larzul C, Lefaucheur L, Ecolan P, Gogue J, Talmant A, Sellier P, et al. Phenotypic and genetic parameters for longissimus muscle fiber characteristics in relation to growth, carcass, and meat quality traits in large white pigs. Journal of Animal Science. 1997;75(12):3126-3137.

9. Kristensen LS, Andersen MS, Stagsted LVW, Ebbesen KK, Hansen TB, Kjems J. The biogenesis, biology and characterization of circular RNAs. Nature Reviews Genetics. 2019.

10. Abdelmohsen K, Panda AC, De S, Grammatikakis I, Kim J, Ding J, et al. Circular RNAs in monkey muscle: age-dependent changes. Aging. 2015;7(11):903-910.

11. Wei X, Li H, Yang J, Hao D, Dong D, Huang Y, et al. Circular RNA profiling reveals an abundant circLMO7 that regulates myoblasts differentiation and survival by sponging miR-378a-3p. Cell Death \& Disease. 2017;8(10):e3153.

12. Legnini I, Di Timoteo G, Rossi F, Morlando M, Briganti F, Sthandier O, et al. Circ-ZNF609 Is a Circular RNA that Can Be Translated and Functions in Myogenesis. Molecular Cell. 2017;66(1):22-37 e29.

13. Liang G, Yang Y, Niu G, Tang Z, Li K. Genome-wide profiling of Sus scrofa circular RNAs across nine organs and three developmental stages. DNA Research. 2017;24(5):523-535.

14. Zhang XO, Wang HB, Zhang Y, Lu X, Chen LL, Yang L. Complementary sequence-mediated exon circularization. Cell. 2014;159(1):134-147.

15. Kim D, Pertea G, Trapnell C, Pimentel H, Kelley R, Salzberg SL. TopHat2: accurate alignment of transcriptomes in the presence of insertions, deletions and gene fusions. Genome Biology. 2013;14(4):R36.

16. Kim D, Salzberg SL. TopHat-Fusion: an algorithm for discovery of novel fusion transcripts. Genome Biology. 2011;12(8):R72.

17. Wang J, Ge J, Cao H, Zhang X, Guo Y, Li X, et al. Leptin Promotes White Adipocyte Browning by Inhibiting the Hh Signaling Pathway. Cells. 2019;8(4).

18. Joo ST, Kim GD, Hwang YH, Ryu YC. Control of fresh meat quality through manipulation of muscle fiber characteristics. Meat Science. 2013;95(4):828-836.

19. Bassel-Duby R, Olson EN. Signaling pathways in skeletal muscle remodeling. Annual Review of Biochemistry. 2006;75:19-37. 
20. Holloszy JO, Coyle EF. Adaptations of skeletal muscle to endurance exercise and their metabolic consequences. J Appl Physiol Respir Environ Exerc Physiol. 1984;56(4):831-838.

21. Hsu MT, Coca-Prados M. Electron microscopic evidence for the circular form of RNA in the cytoplasm of eukaryotic cells. Nature. 1979;280(5720):339-340.

22. Huang M, Shen $Y$, Mao H, Chen L, Chen J, Guo X, et al. Circular RNA expression profiles in the porcine liver of two distinct phenotype pig breeds. Asian-Australasian Journal of Animal Sciences. 2018;31(6):812-819.

23. Sun J, Xie M, Huang Z, Li H, Chen T, Sun R, et al. Integrated analysis of non-coding RNA and mRNA expression profiles of 2 pig breeds differing in muscle traits. Journal of Animal Science. 2017;95(3):1092-1103.

24. Shen L, Gan M, Tang Q, Tang G, Jiang Y, Li M, et al. Comprehensive Analysis of IncRNAs and circRNAs Reveals the Metabolic Specialization in Oxidative and Glycolytic Skeletal Muscles. International Journal of Molecular Sciences. 2019;20(12).

25. Veno MT, Hansen TB, Veno ST, Clausen BH, Grebing M, Finsen B, et al. Spatio-temporal regulation of circular RNA expression during porcine embryonic brain development. Genome Biology. 2015;16:245.

26. Jeck WR, Sorrentino JA, Wang K, Slevin MK, Burd CE, Liu J, et al. Circular RNAs are abundant, conserved, and associated with ALU repeats. RNA. 2013;19(2):141-157.

27. Liang D, Wilusz JE. Short intronic repeat sequences facilitate circular RNA production. Genes and Development. 2014;28(20):2233-2247.

28. Wang T, Xu YQ, Yuan YX, Xu PW, Zhang C, Li F, et al. Succinate induces skeletal muscle fiber remodeling via SUNCR1 signaling pathway. EMBO reports. 2019:e47892.

29. Chen X, Guo Y, Jia G, Zhao H, Liu G, Huang Z. Ferulic acid regulates muscle fiber type formation through the Sirt1/AMPK signaling pathway. Food \& Function. 2019;10(1):259-265.

30. Liu L, Yao L, Wang S, Chen Z, Han T, Ma P, et al. 6-Gingerol Improves Ectopic Lipid Accumulation, Mitochondrial Dysfunction, and Insulin Resistance in Skeletal Muscle of Ageing Rats: Dual Stimulation of the AMPK/PGC-1alpha Signaling Pathway via Plasma Adiponectin and Muscular AdipoR1. Molecular Nutrition \& Food Research. 2019;63(6):e1800649.

31. Kitamura T, Kitamura YI, Funahashi Y, Shawber CJ, Castrillon DH, Kollipara R, et al. A Foxo/Notch pathway controls myogenic differentiation and fiber type specification. Journal of Clinical Investigation. 2007;117(9):2477-2485.

32. Stitt TN, Drujan D, Clarke BA, Panaro F, Timofeyva Y, Kline WO, et al. The IGF-1/PI3K/Akt pathway prevents expression of muscle atrophy-induced ubiquitin ligases by inhibiting FOXO transcription factors. Molecular Cell. 2004;14(3):395-403.

33. Yu M, Wang H, Xu Y, Yu D, Li D, Liu X, et al. Insulin-like growth factor-1 (IGF-1) promotes myoblast proliferation and skeletal muscle growth of embryonic chickens via the PI3K/Akt signalling pathway. Cell Biology International. 2015;39(8):910-922.

34. Zhong Z, Huang M, Lv M, He Y, Duan C, Zhang L, et al. Circular RNA MYLK as a competing endogenous RNA promotes bladder cancer progression through modulating VEGFA/VEGFR2 


\section{Figures}

A

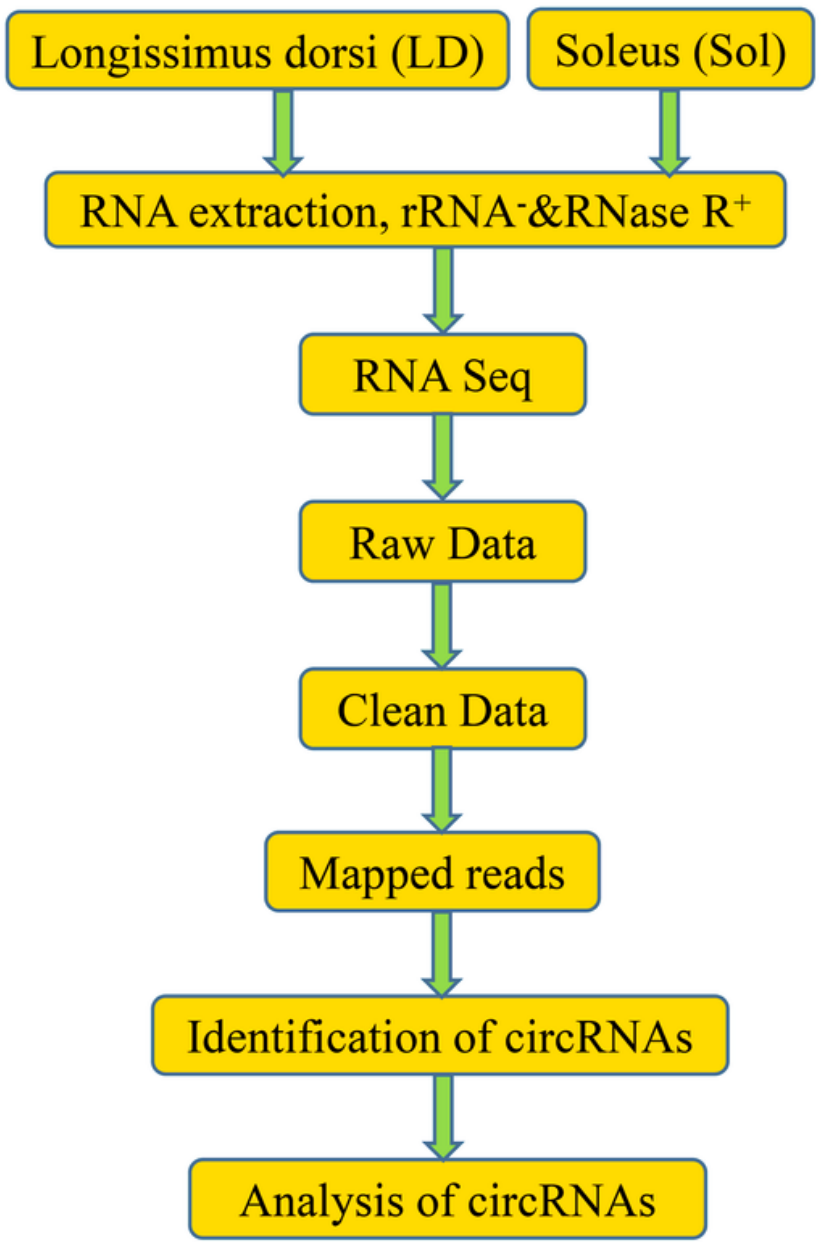

B

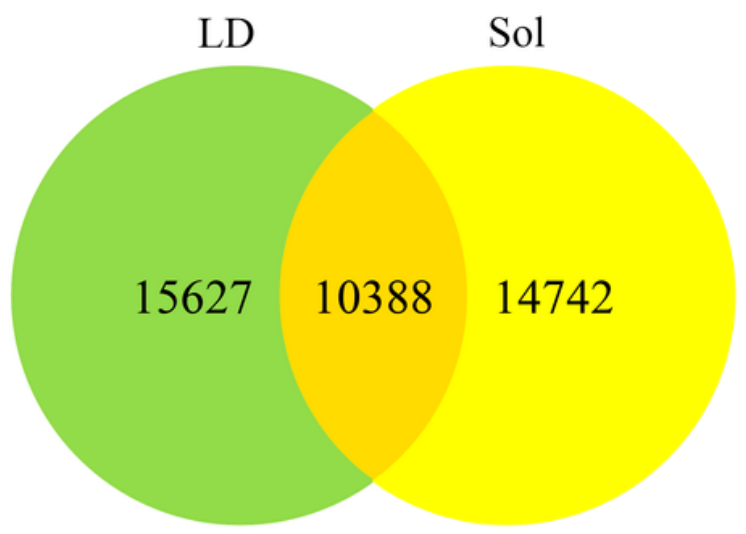

$\mathrm{C}$

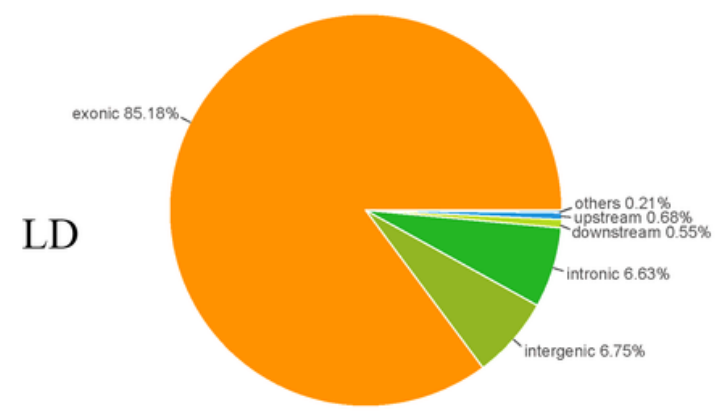

Sol

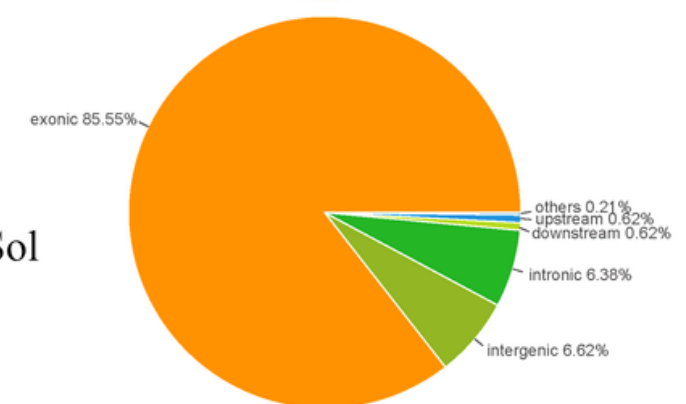

\section{Figure 1}

Identification of circular RNAs in LD and Sol skeletal muscles. (A) Pipeline for circRNA identification. (B) Venn diagram showing the overlap of annotated circRNAs among LD and Sol skeletal muscles. (C) Origin of circRNAs described in this study in the Sus scrofa genome. 
A

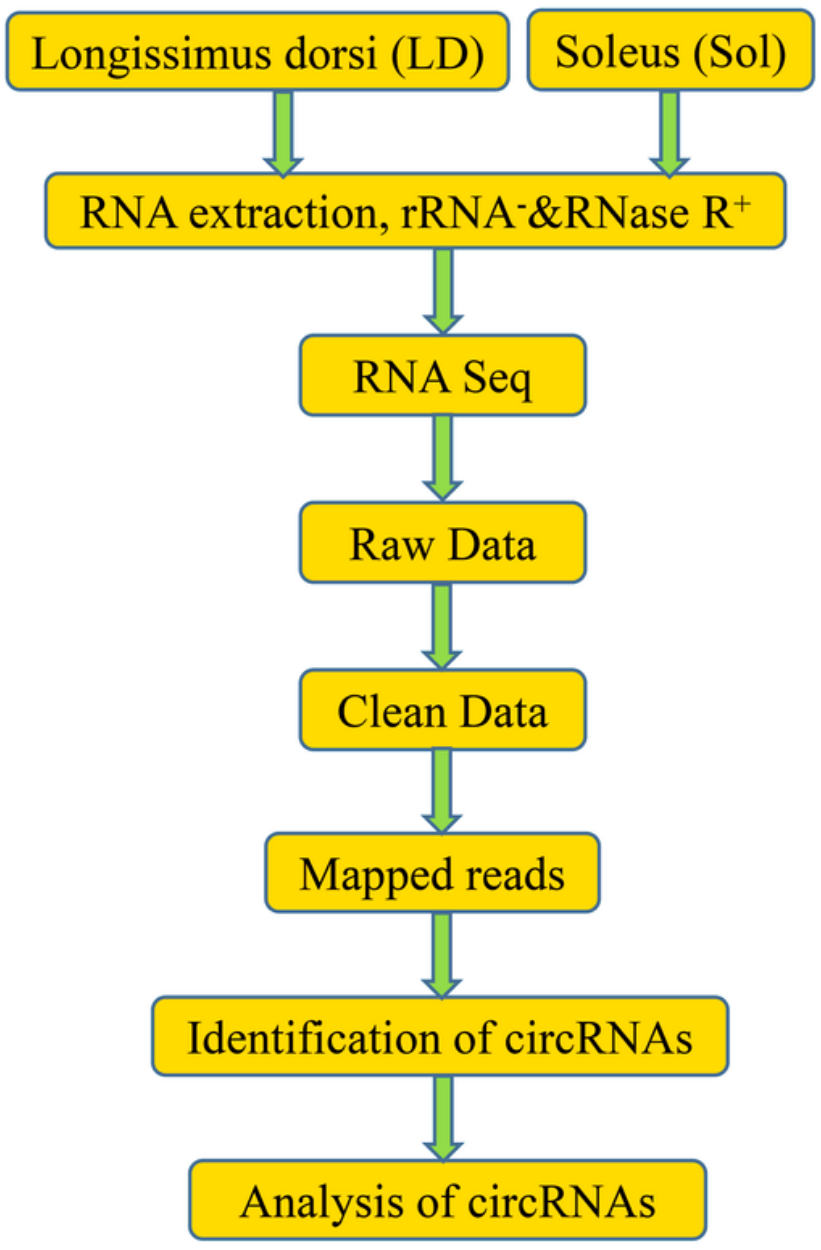

B

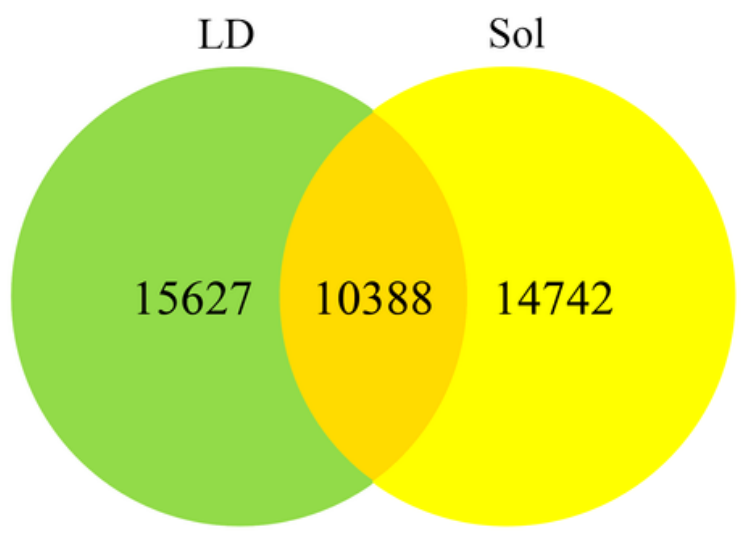

$\mathrm{C}$

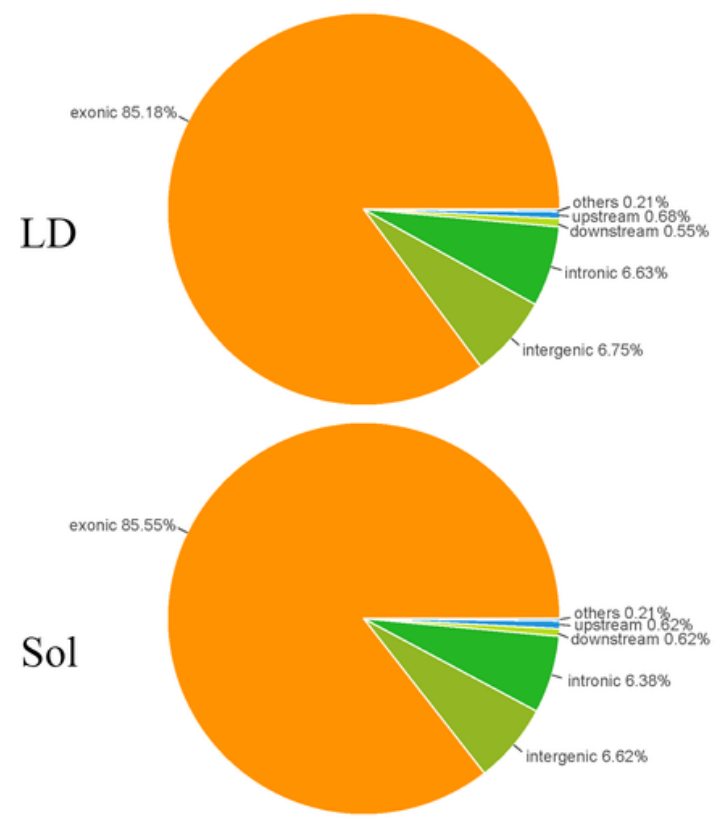

\section{Figure 1}

Identification of circular RNAs in LD and Sol skeletal muscles. (A) Pipeline for circRNA identification. (B) Venn diagram showing the overlap of annotated circRNAs among LD and Sol skeletal muscles. (C) Origin of circRNAs described in this study in the Sus scrofa genome. 
A

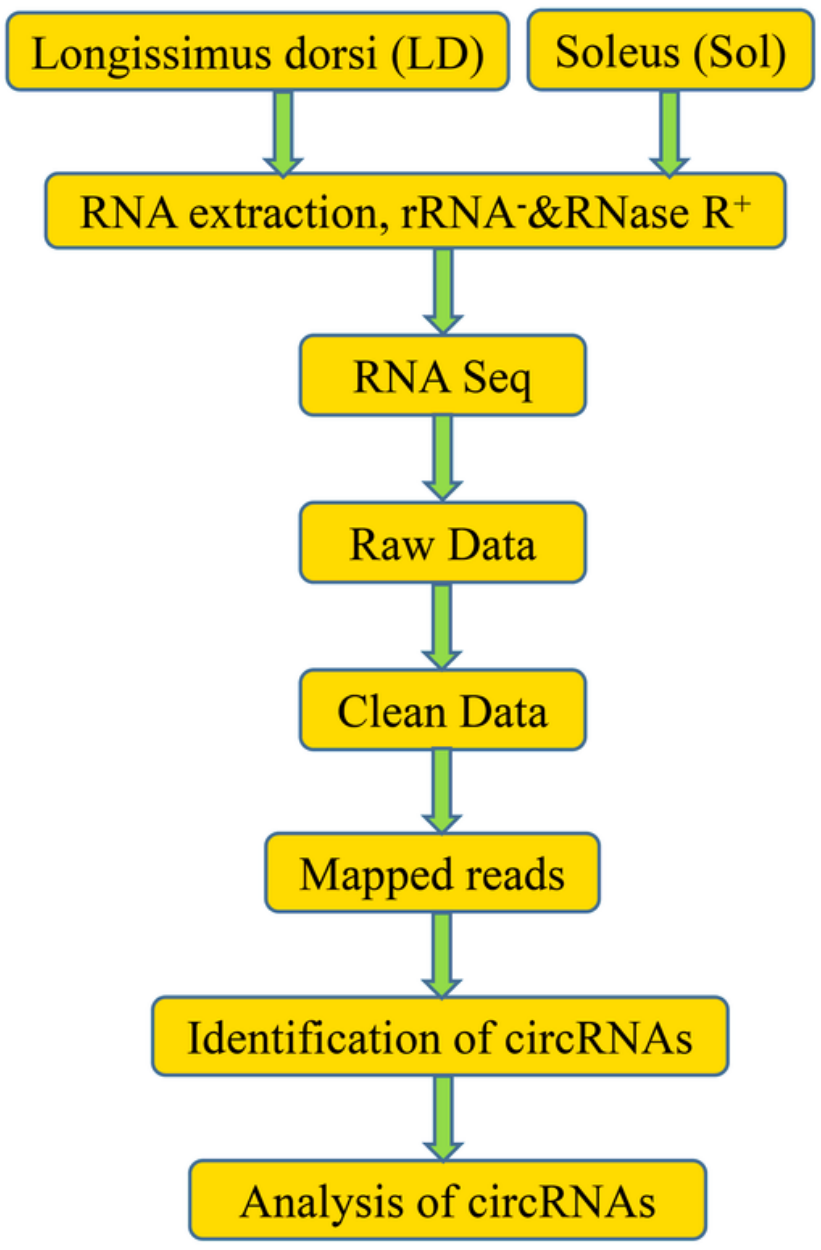

B

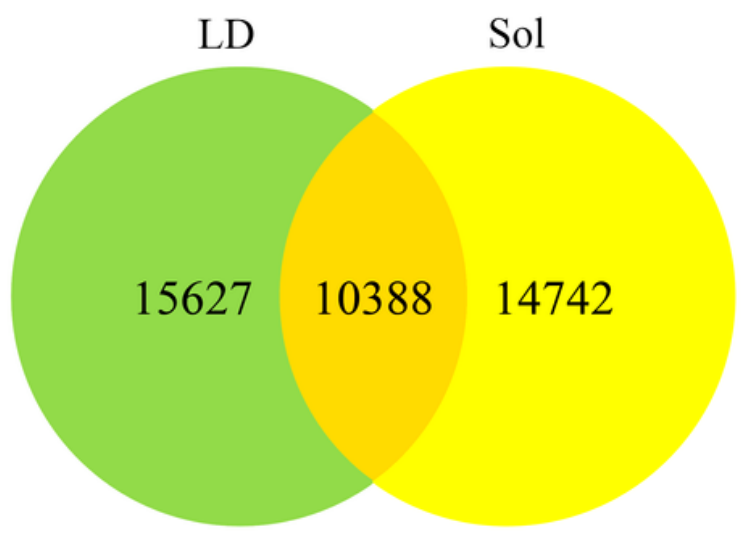

$\mathrm{C}$

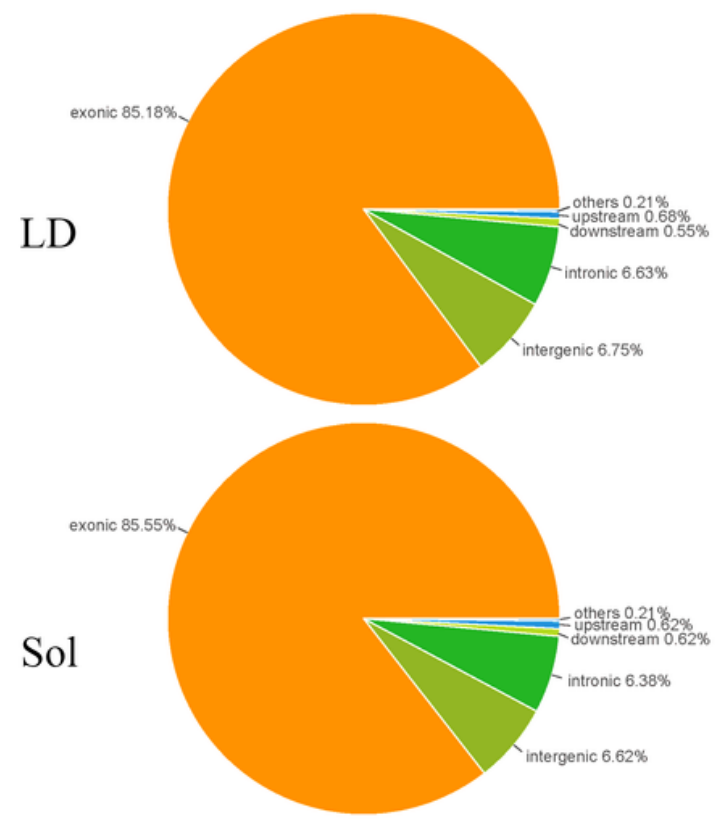

\section{Figure 1}

Identification of circular RNAs in LD and Sol skeletal muscles. (A) Pipeline for circRNA identification. (B) Venn diagram showing the overlap of annotated circRNAs among LD and Sol skeletal muscles. (C) Origin of circRNAs described in this study in the Sus scrofa genome. 
A

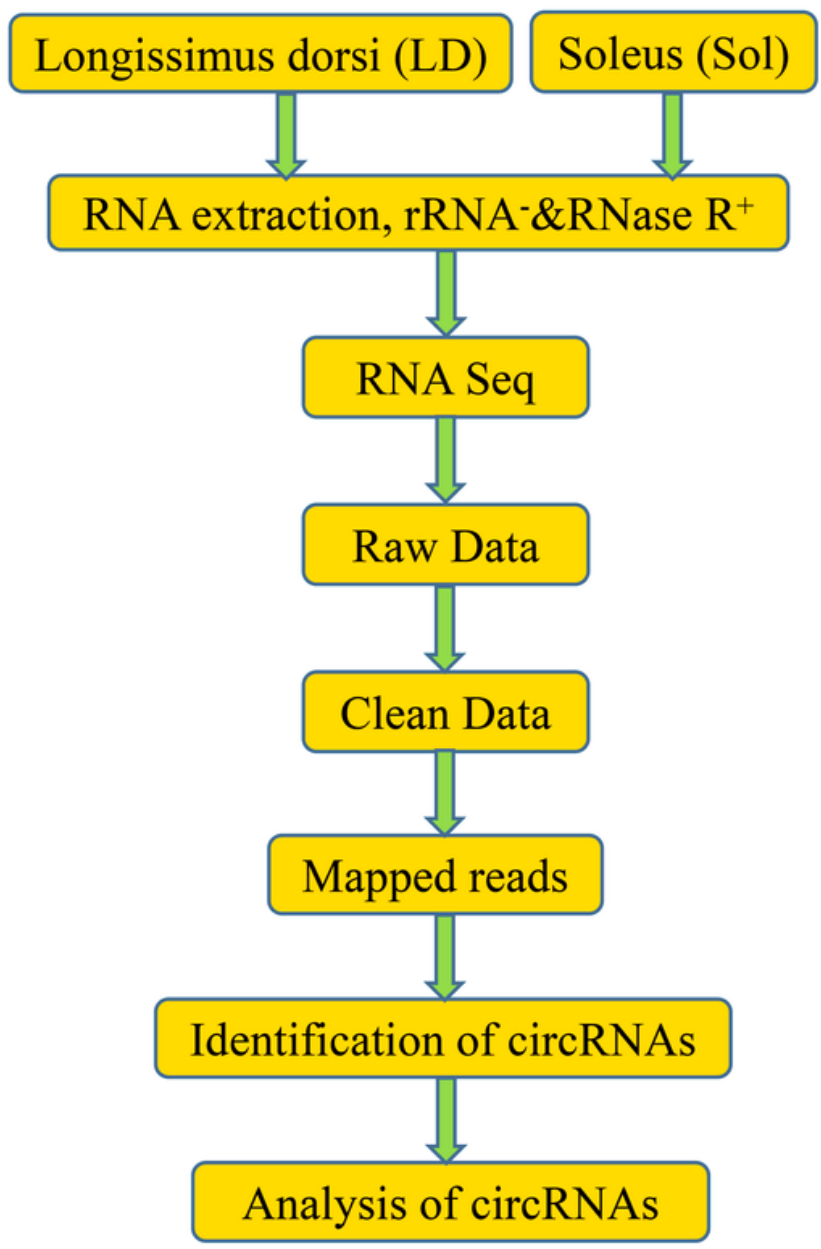

B

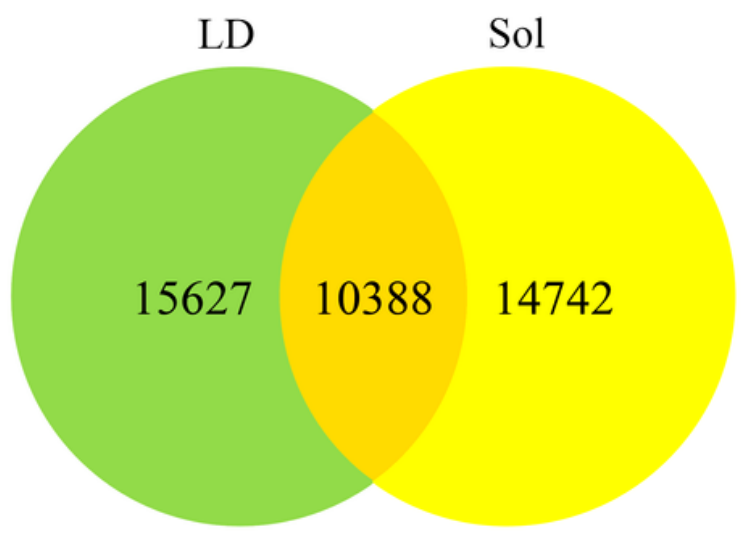

C

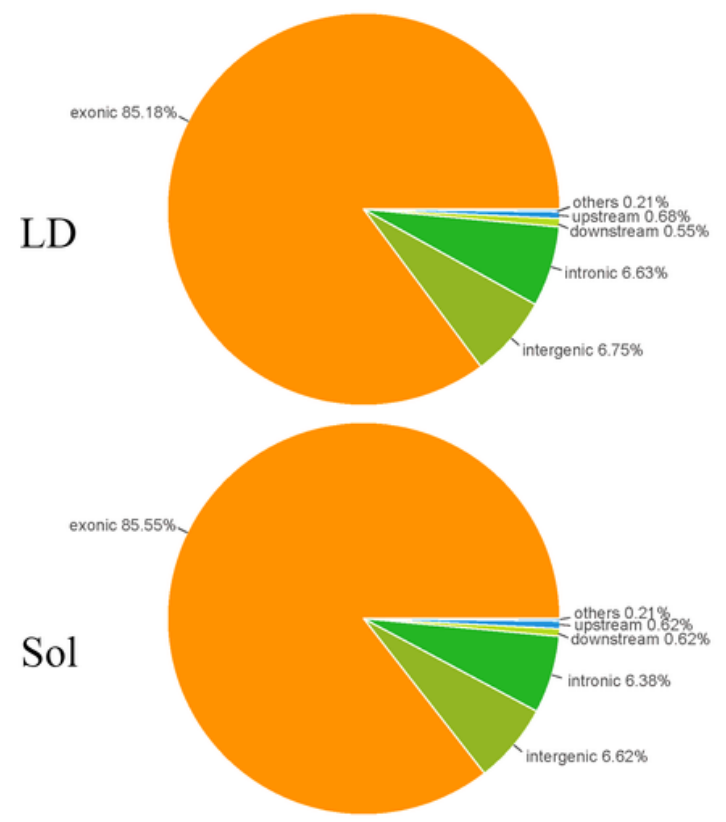

\section{Figure 2}

Identification of circular RNAs in LD and Sol skeletal muscles. (A) Pipeline for circRNA identification. (B) Venn diagram showing the overlap of annotated circRNAs among LD and Sol skeletal muscles. (C) Origin of circRNAs described in this study in the Sus scrofa genome. 
A

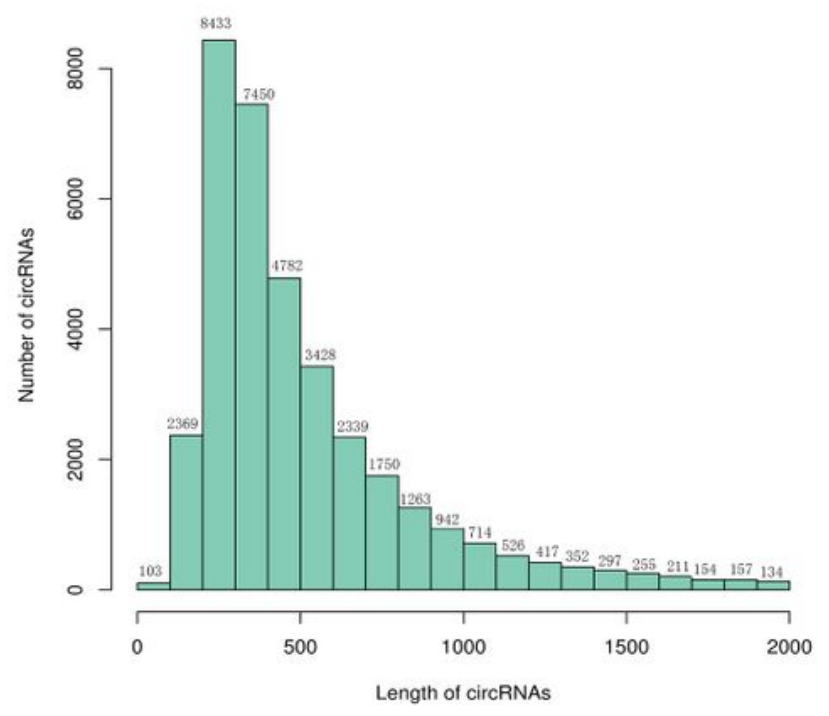

C

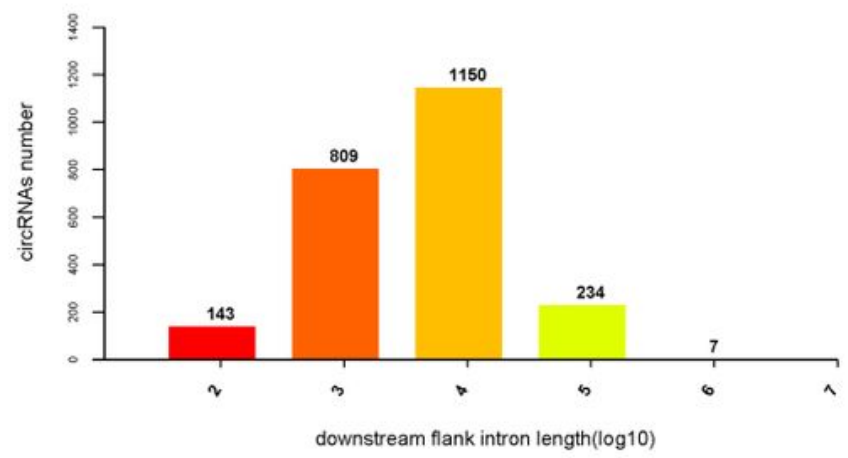

E

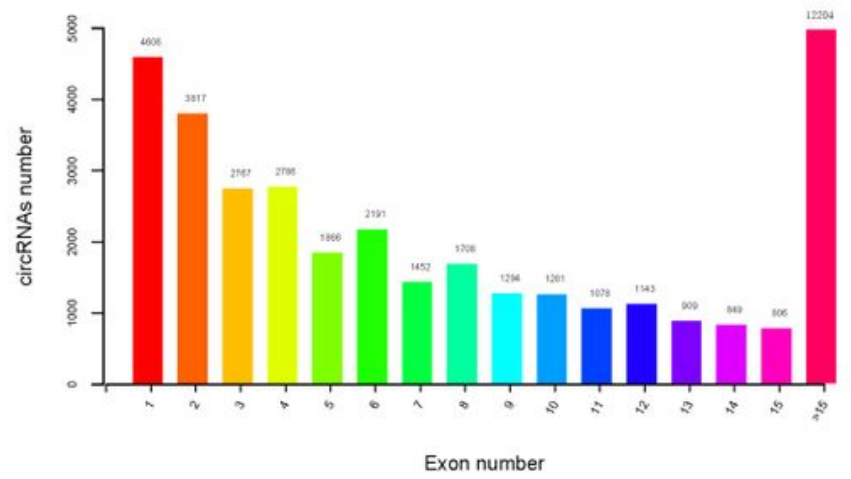

B

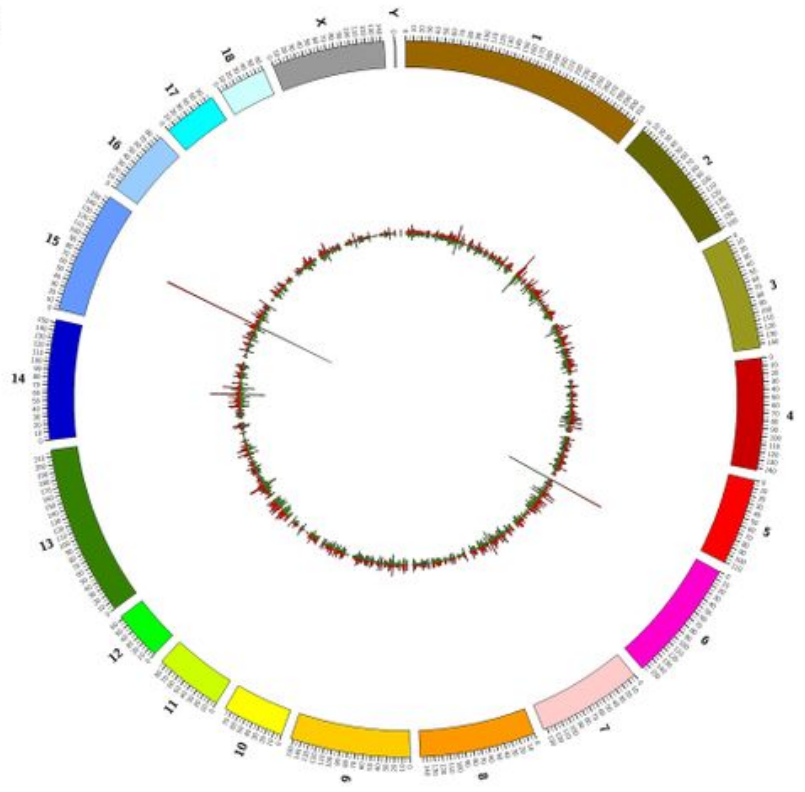

D

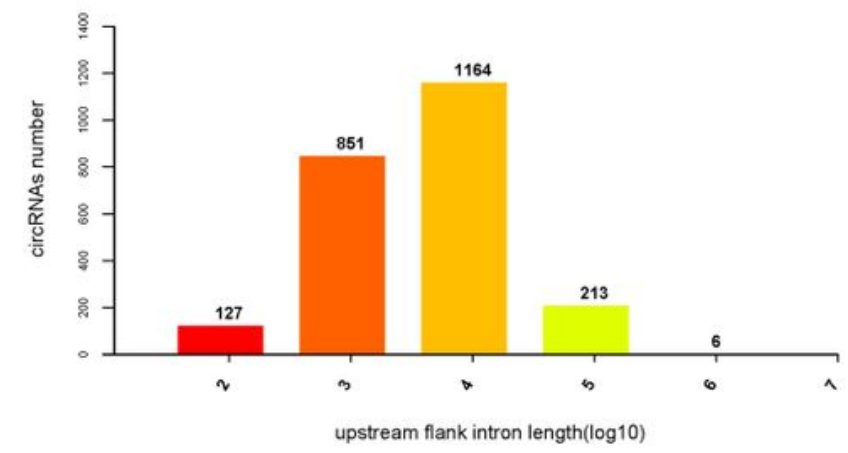

F

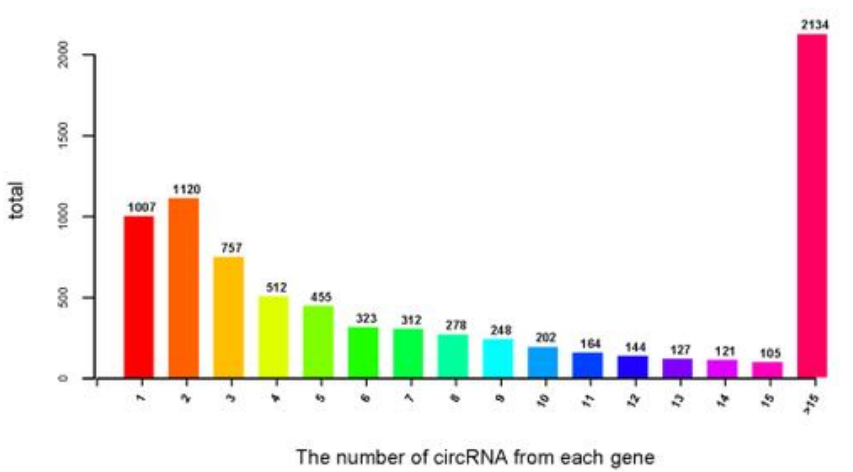

Figure 2

General characteristics of circular RNAs in pigs. (A) Length distribution of circRNAs. (B) Circos plot showing the distribution of circRNAs on different chromosomes. (C-D) Length of circRNAs flanking introns. (E) circRNAs that contained varying numbers of exons. (F) Distribution of the number of circRNAs per gene. 
A

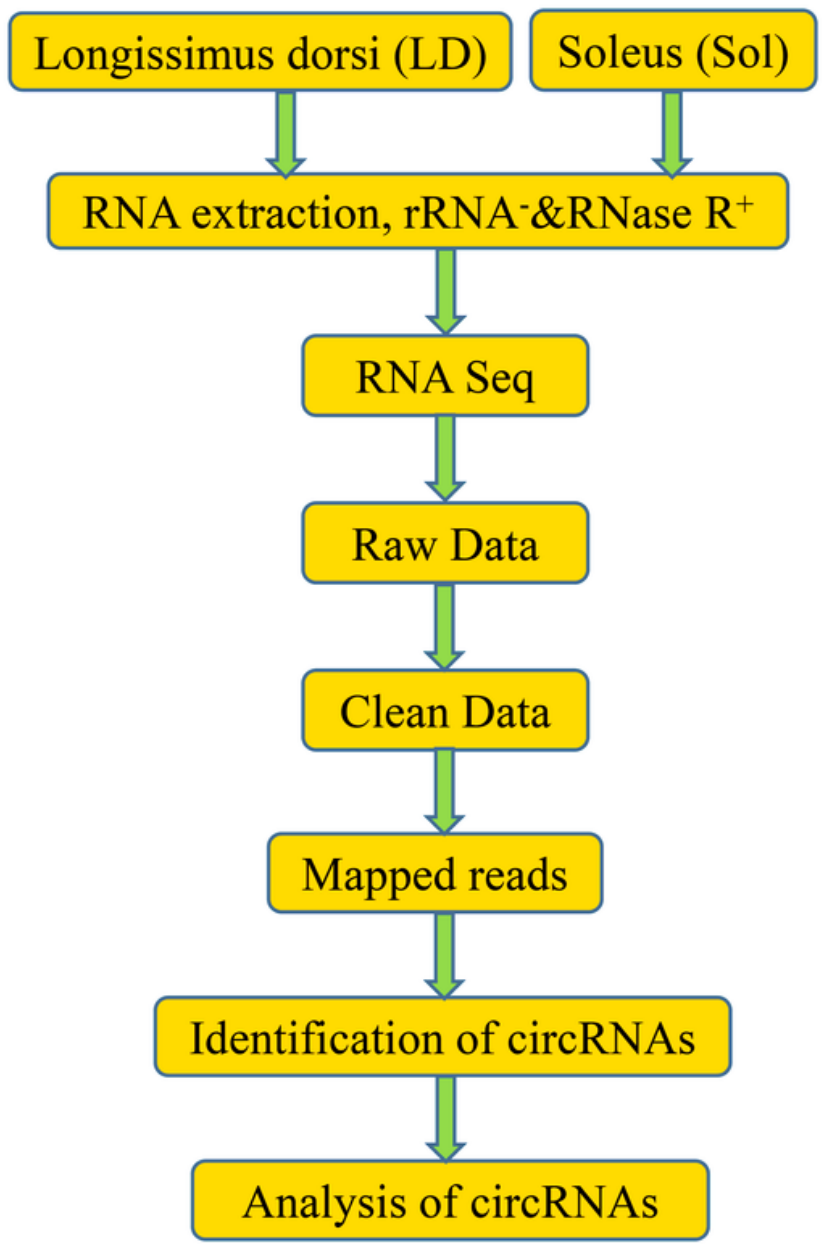

B

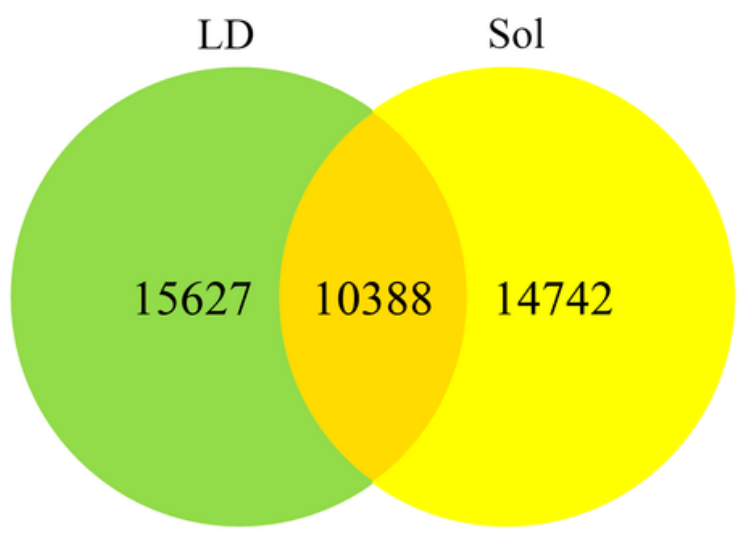

C

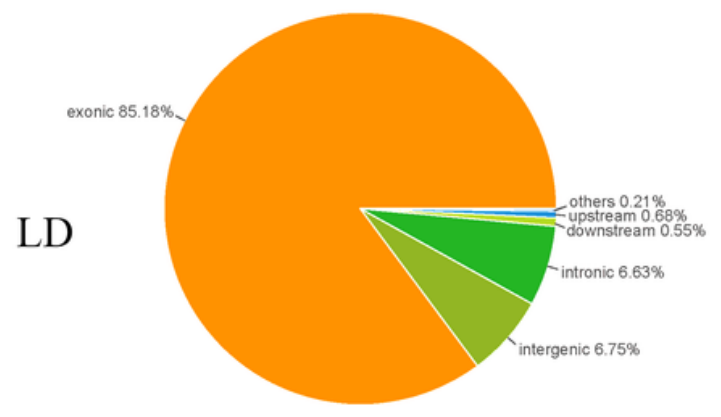

Sol

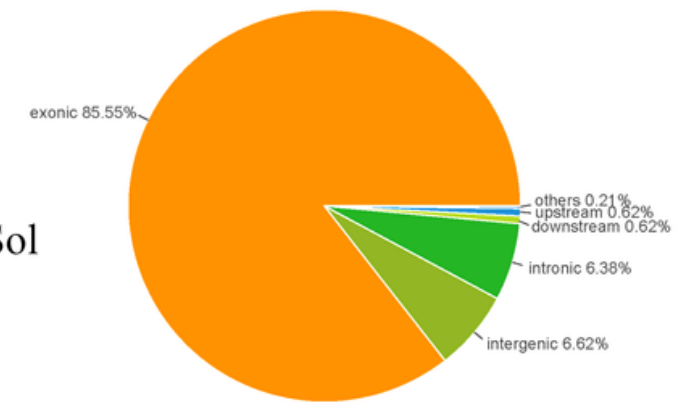

\section{Figure 2}

Identification of circular RNAs in LD and Sol skeletal muscles. (A) Pipeline for circRNA identification. (B) Venn diagram showing the overlap of annotated circRNAs among LD and Sol skeletal muscles. (C) Origin of circRNAs described in this study in the Sus scrofa genome. 
A

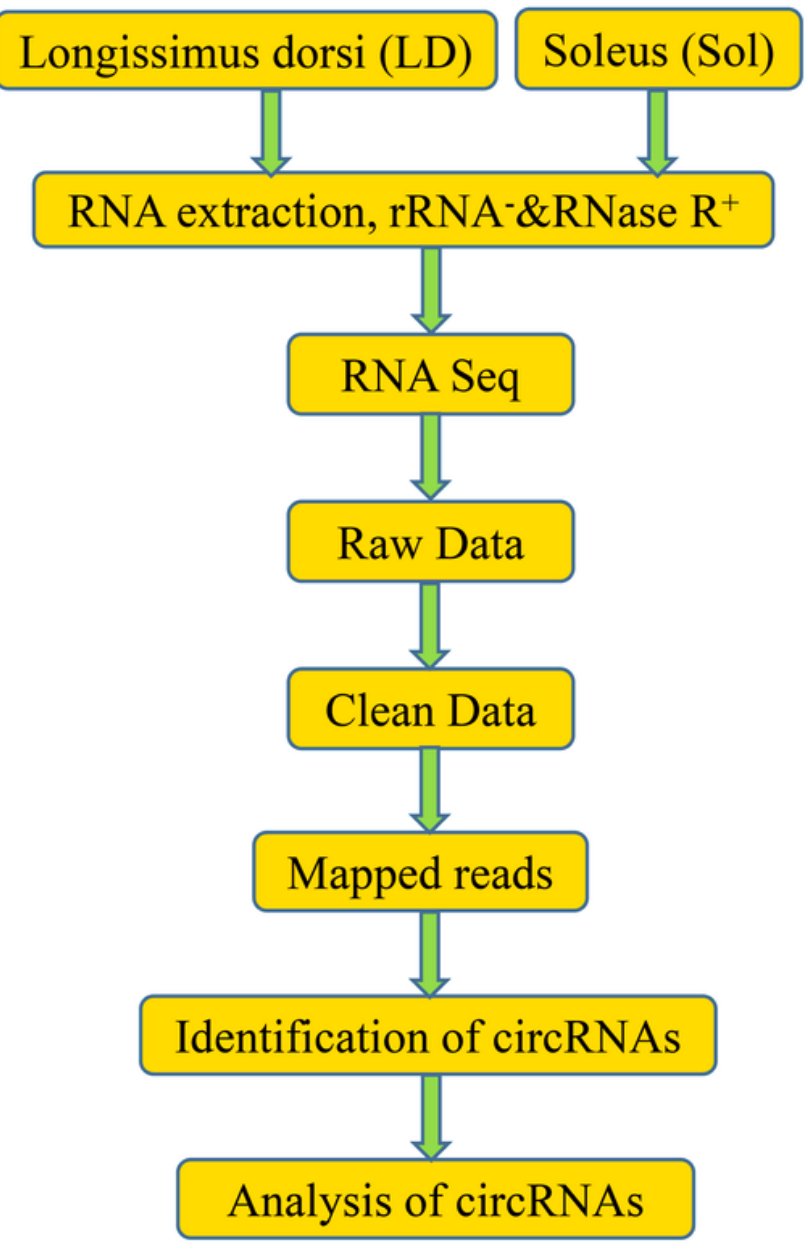

B

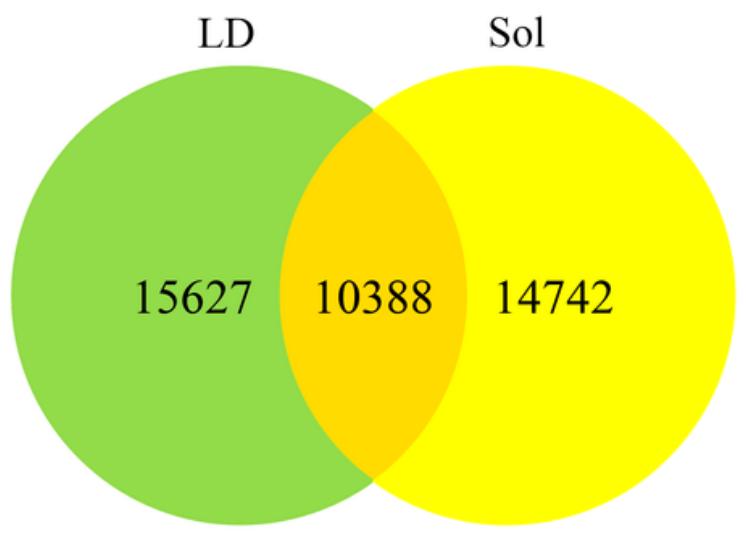

$\mathrm{C}$

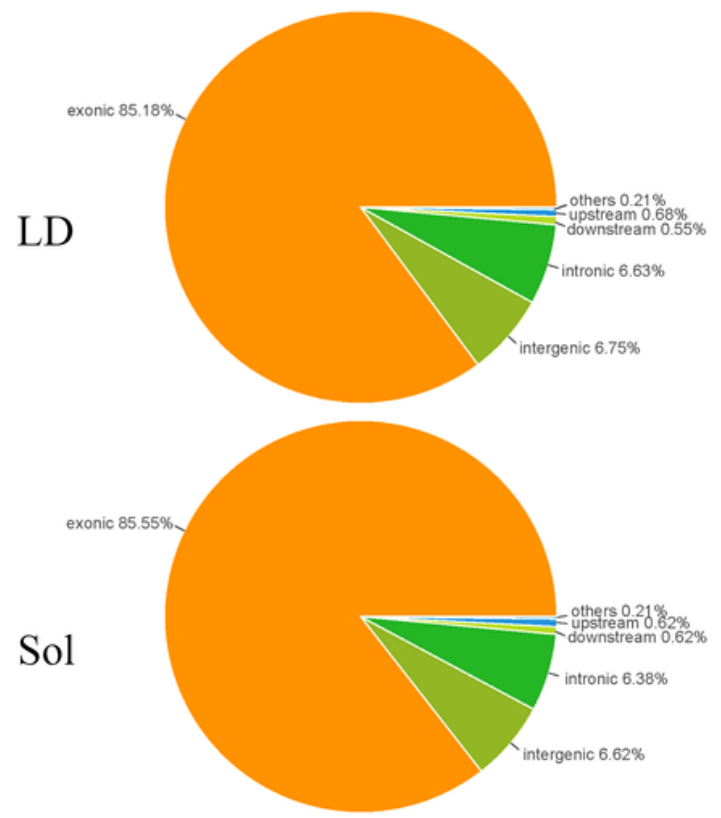

\section{Figure 2}

Identification of circular RNAs in LD and Sol skeletal muscles. (A) Pipeline for circRNA identification. (B) Venn diagram showing the overlap of annotated circRNAs among LD and Sol skeletal muscles. (C) Origin of circRNAs described in this study in the Sus scrofa genome. 
A

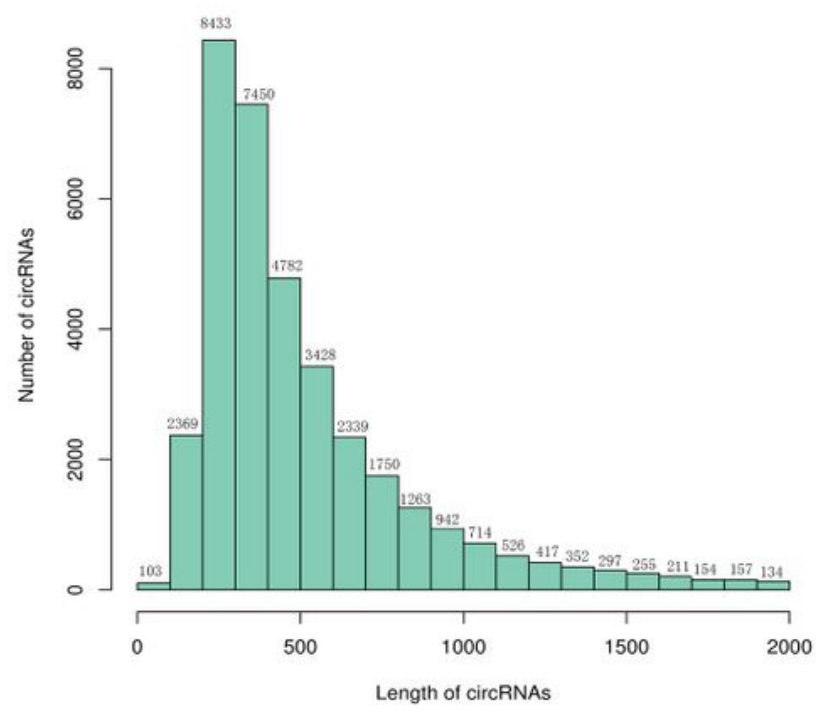

C

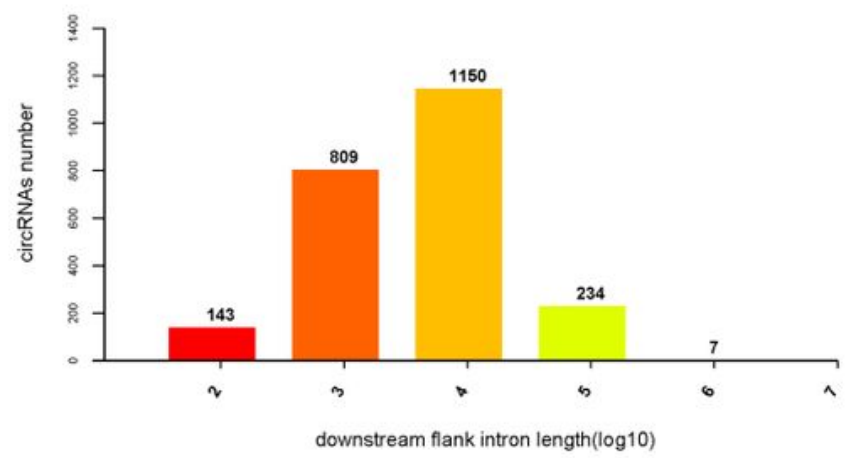

E

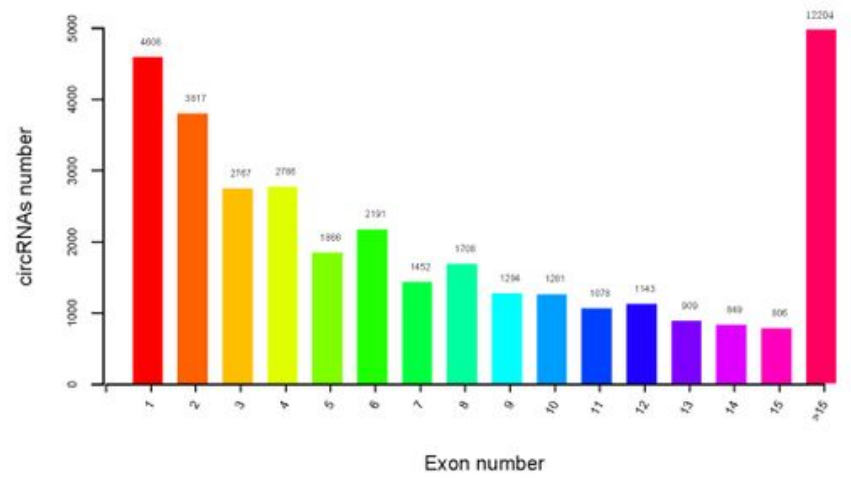

B

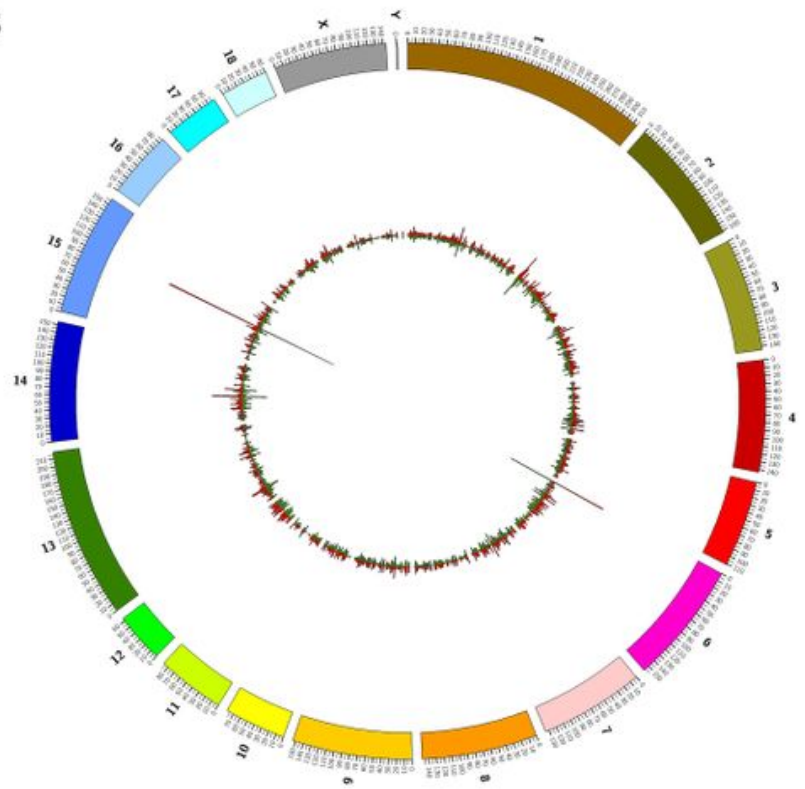

D

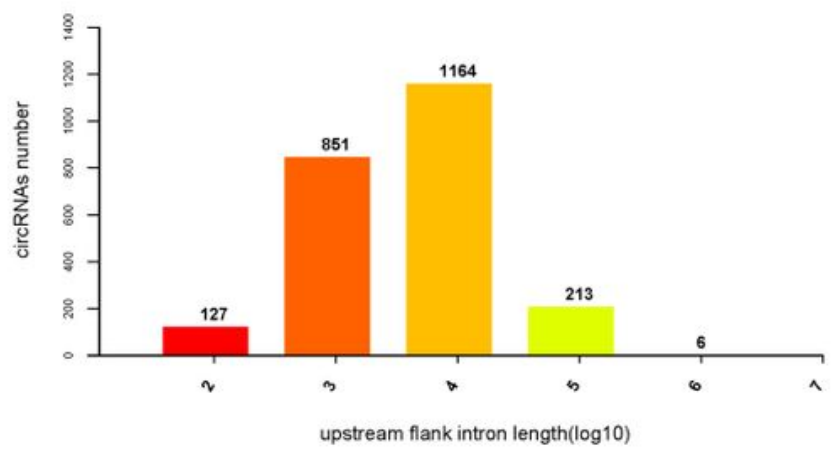

F

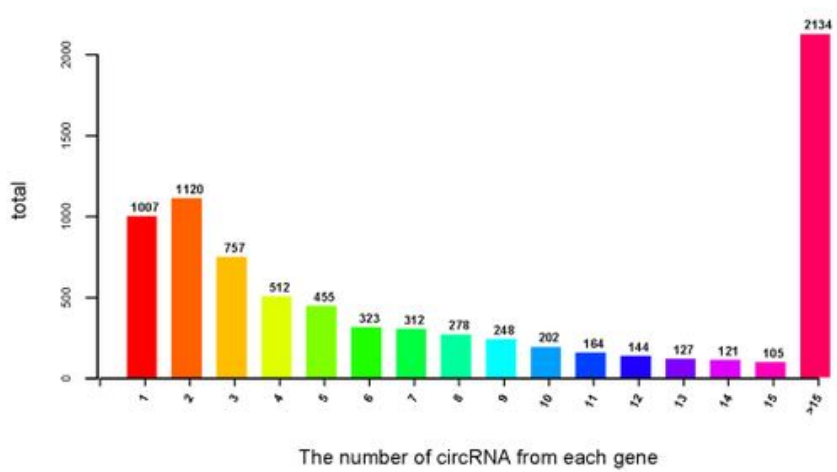

Figure 2

General characteristics of circular RNAs in pigs. (A) Length distribution of circRNAs. (B) Circos plot showing the distribution of circRNAs on different chromosomes. (C-D) Length of circRNAs flanking introns. (E) circRNAs that contained varying numbers of exons. (F) Distribution of the number of circRNAs per gene. 
A

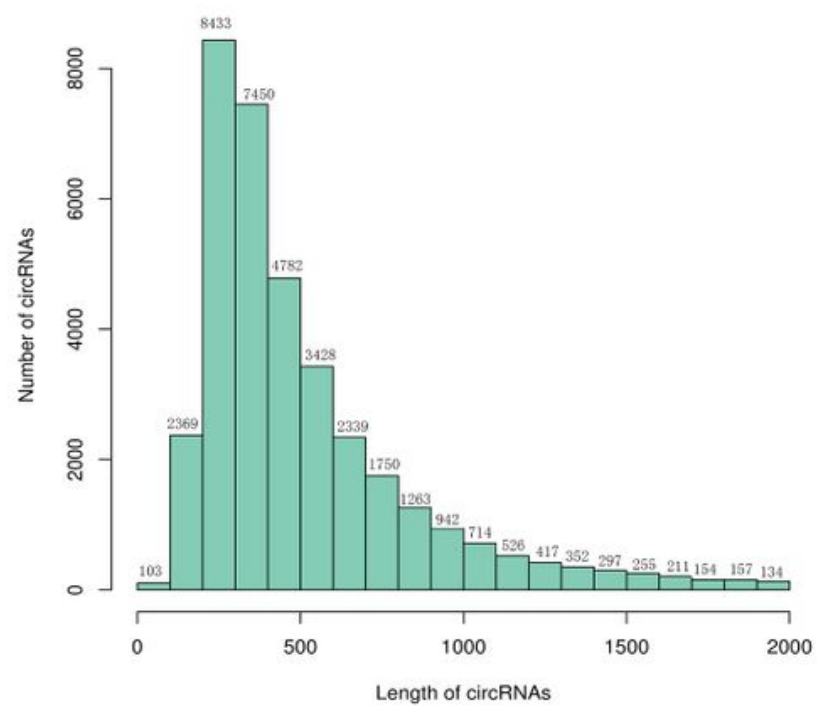

C

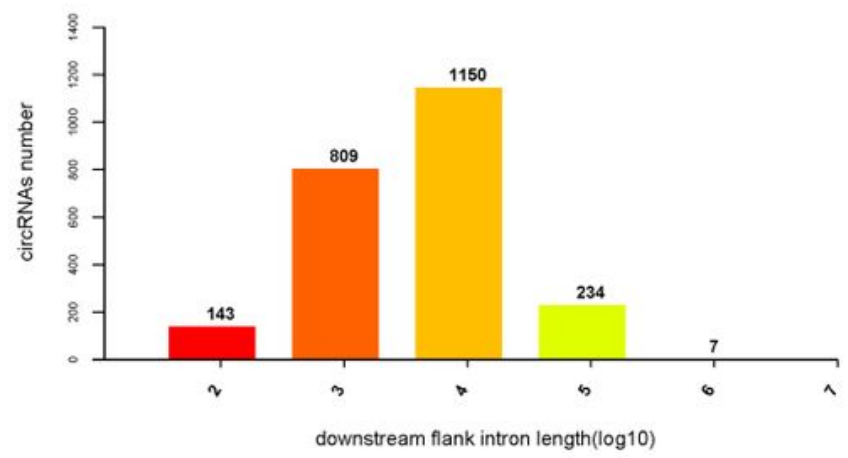

E

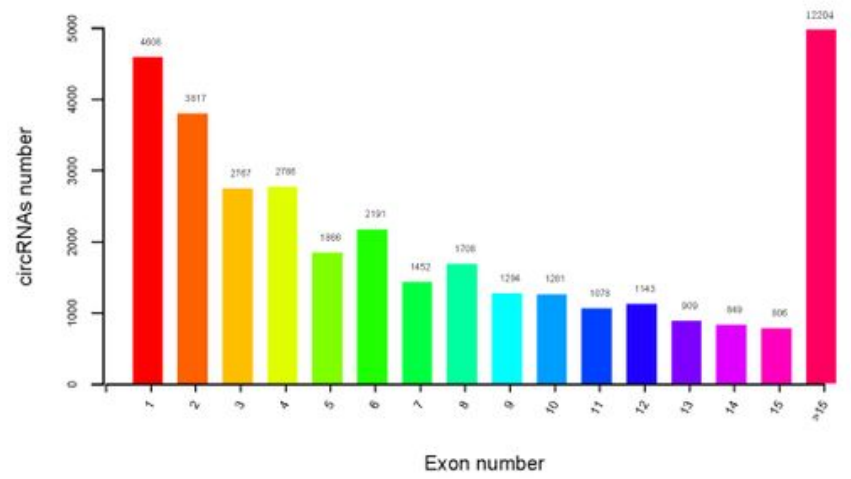

B

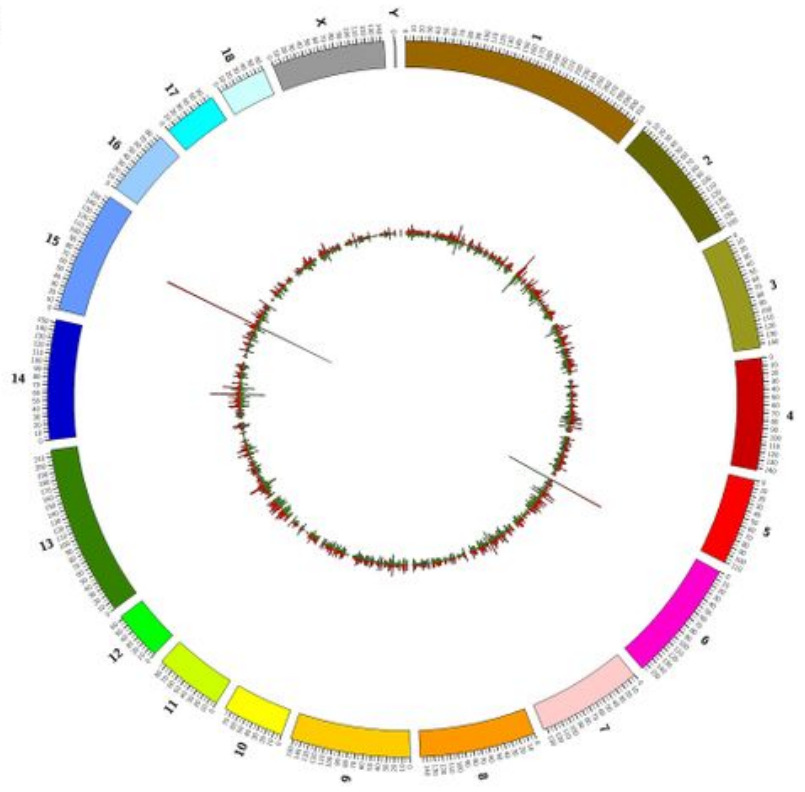

D

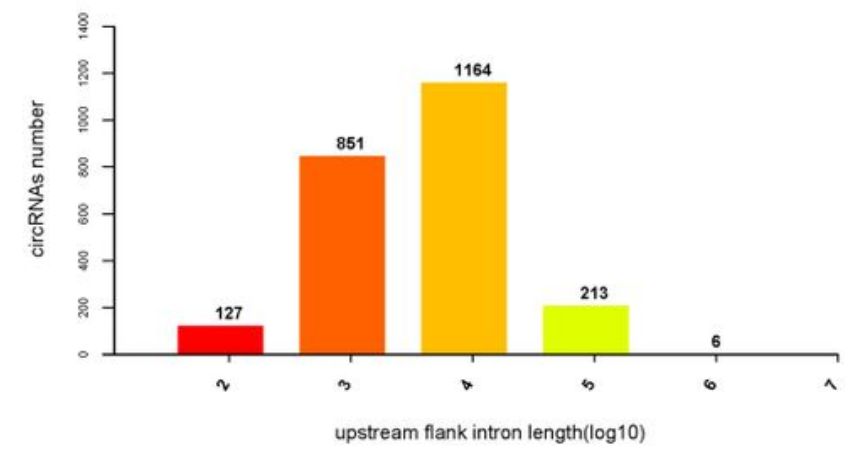

F

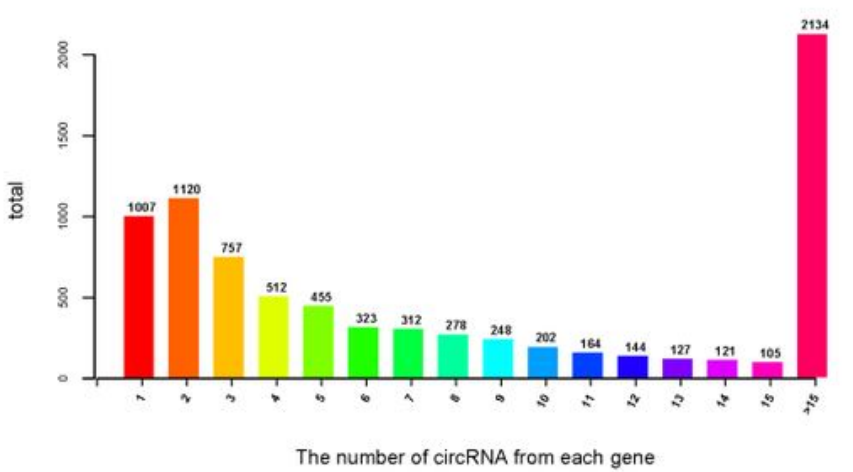

Figure 3

General characteristics of circular RNAs in pigs. (A) Length distribution of circRNAs. (B) Circos plot showing the distribution of circRNAs on different chromosomes. (C-D) Length of circRNAs flanking introns. (E) circRNAs that contained varying numbers of exons. (F) Distribution of the number of circRNAs per gene. 
A

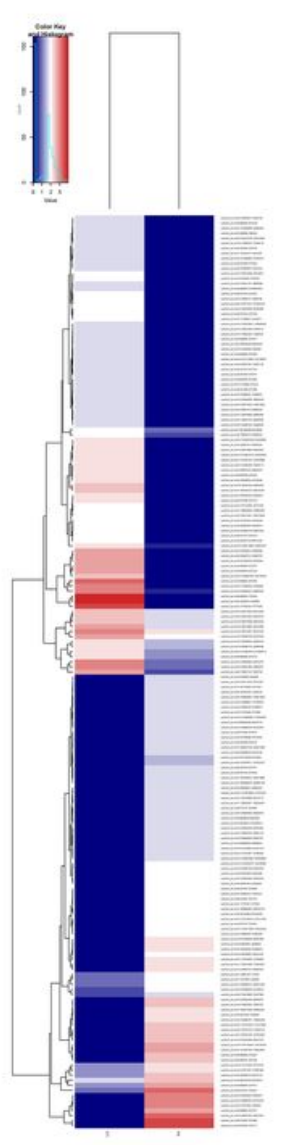

B

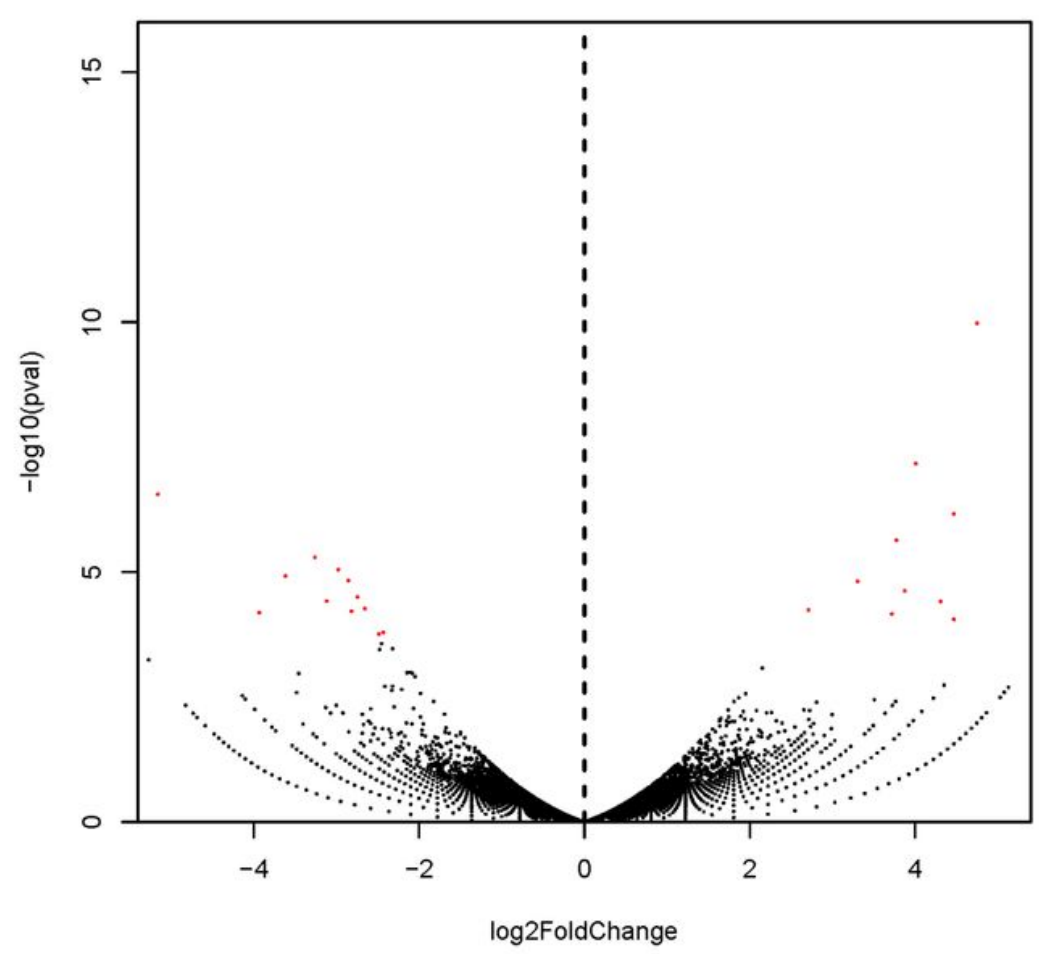

Figure 3

Differential circular RNA expression between LD and Sol skeletal muscles. (A) Cluster heat-map of differentially expressed circRNAs from each sample. (B) Volcano plots showing -log10 (pval) versus log2 fold difference in circRNAs abundance in RPM between LD and Sol skeletal muscles. Red dots denote significantly differently expressed circRNAs. 
A
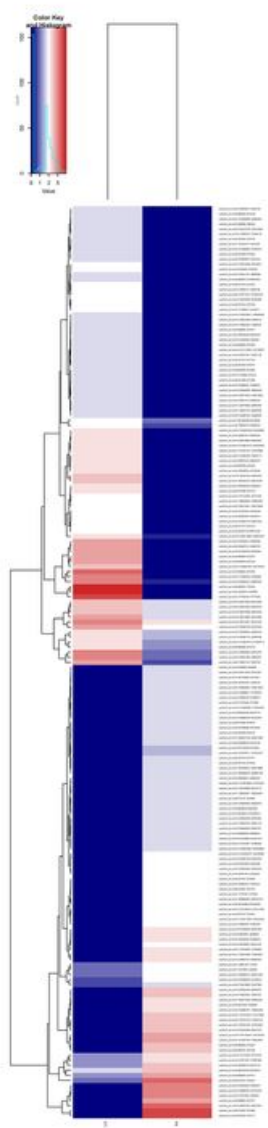

B

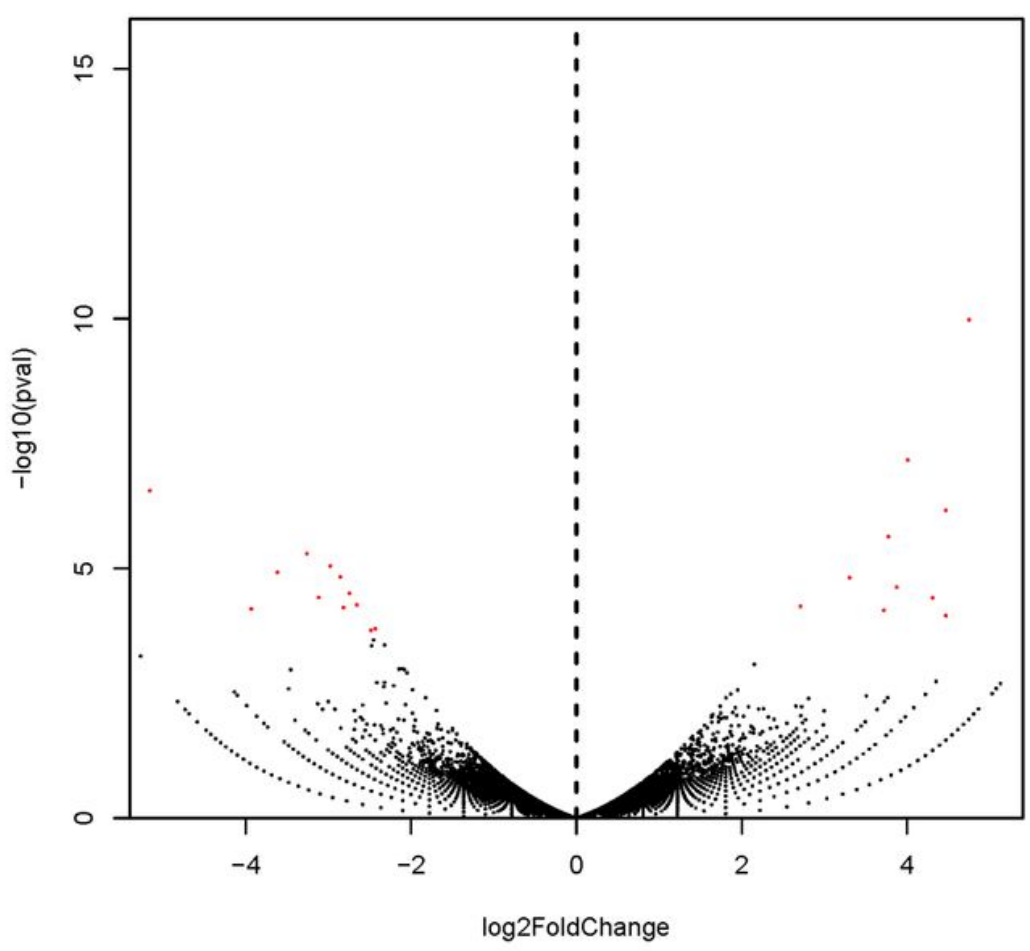

Figure 4

Differential circular RNA expression between LD and Sol skeletal muscles. (A) Cluster heat-map of differentially expressed circRNAs from each sample. (B) Volcano plots showing -log10 (pval) versus log2 fold difference in circRNAs abundance in RPM between LD and Sol skeletal muscles. Red dots denote significantly differently expressed circRNAs. 


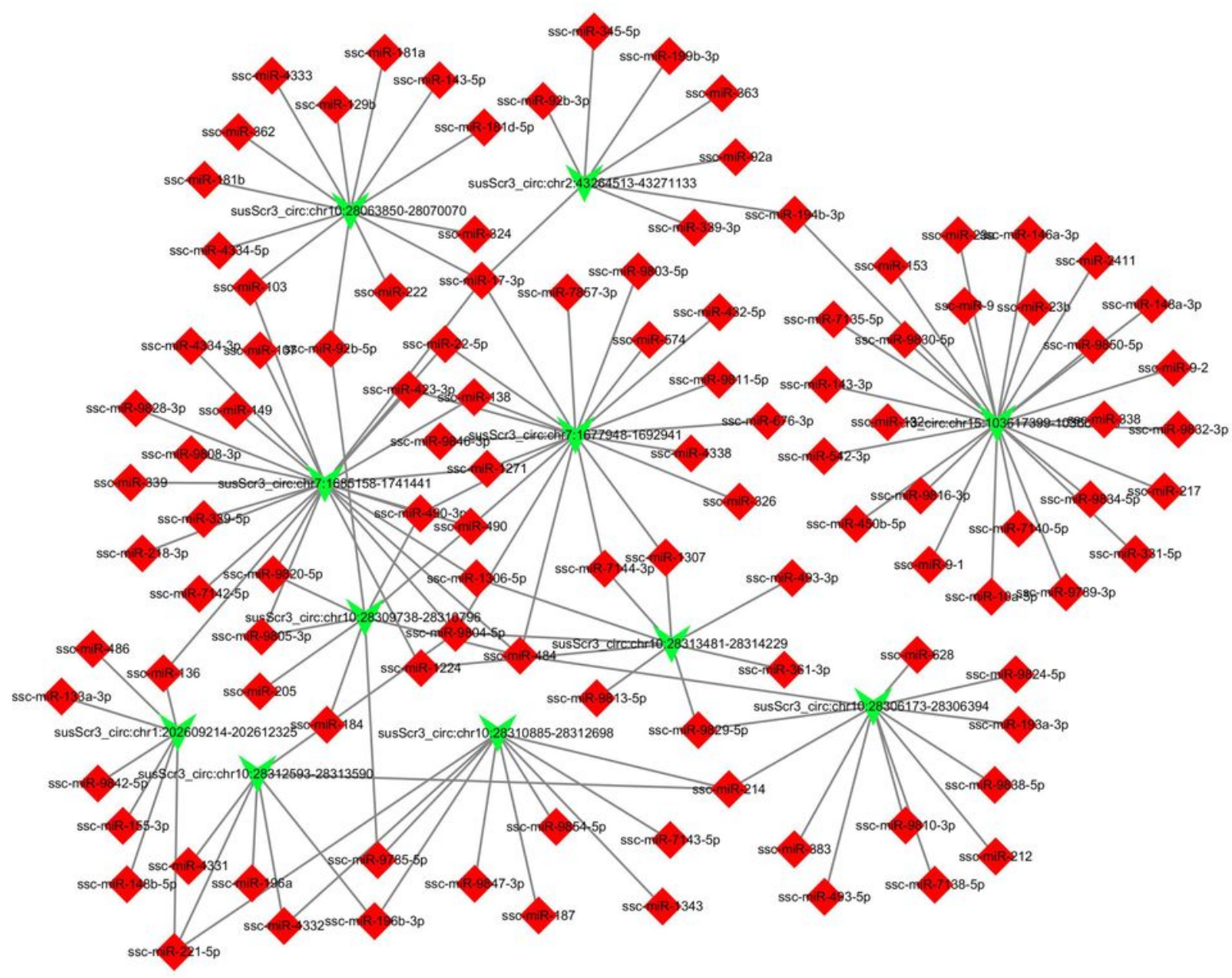

Figure 4

Co-expression network of the miRNAs and candidate circRNAs. Red squares represent miRNAs. Green arrows represent circRNAs. 
A

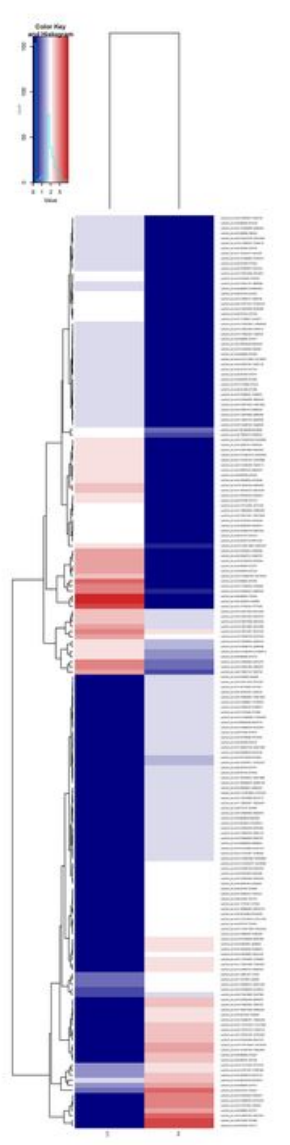

B

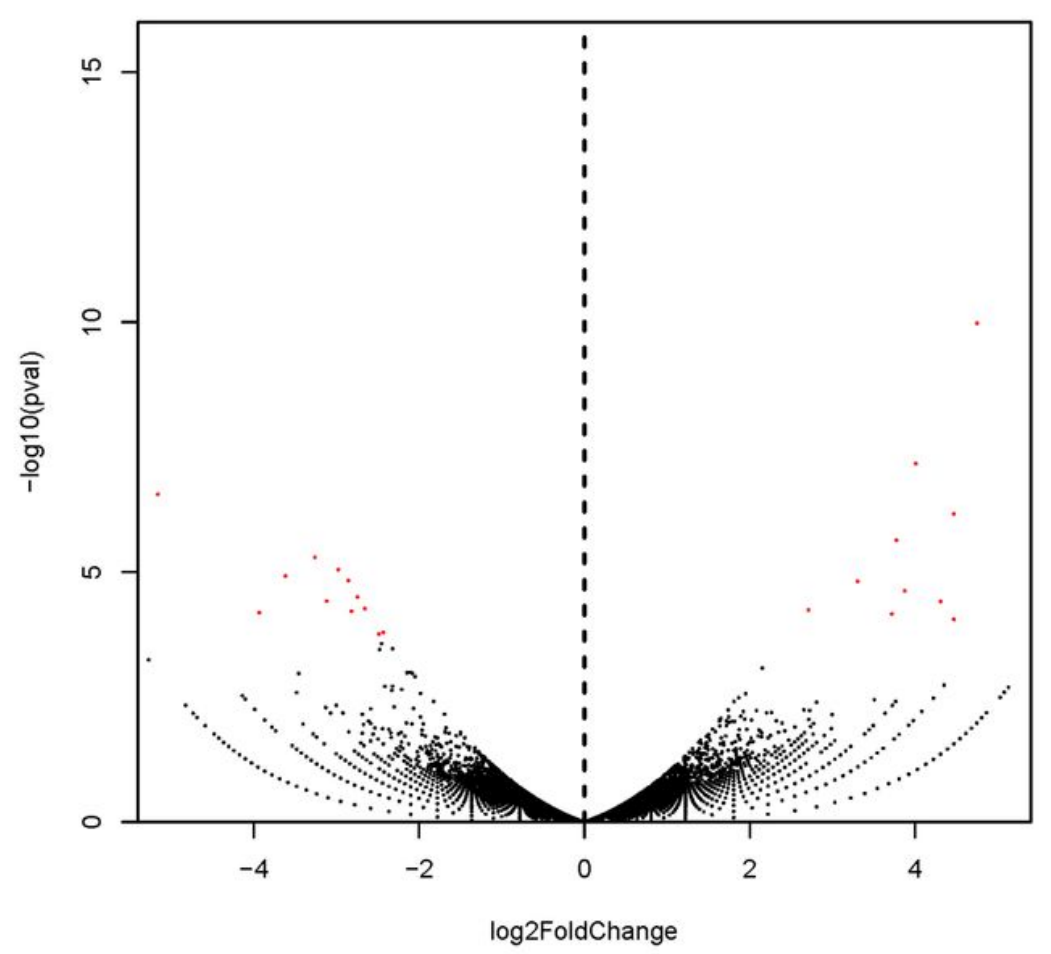

Figure 4

Differential circular RNA expression between LD and Sol skeletal muscles. (A) Cluster heat-map of differentially expressed circRNAs from each sample. (B) Volcano plots showing -log10 (pval) versus log2 fold difference in circRNAs abundance in RPM between LD and Sol skeletal muscles. Red dots denote significantly differently expressed circRNAs. 


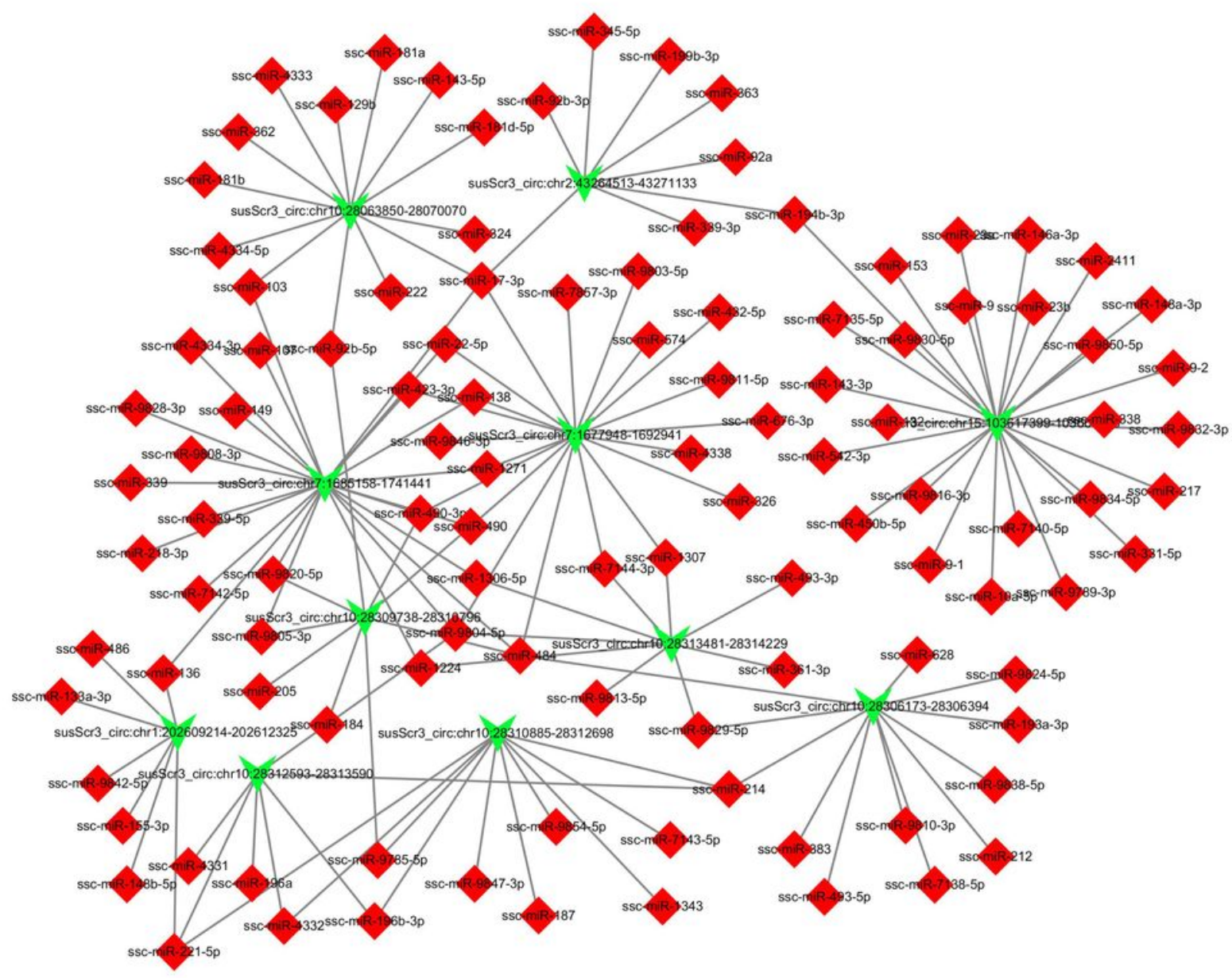

Figure 5

Co-expression network of the miRNAs and candidate circRNAs. Red squares represent miRNAs. Green arrows represent circRNAs. 


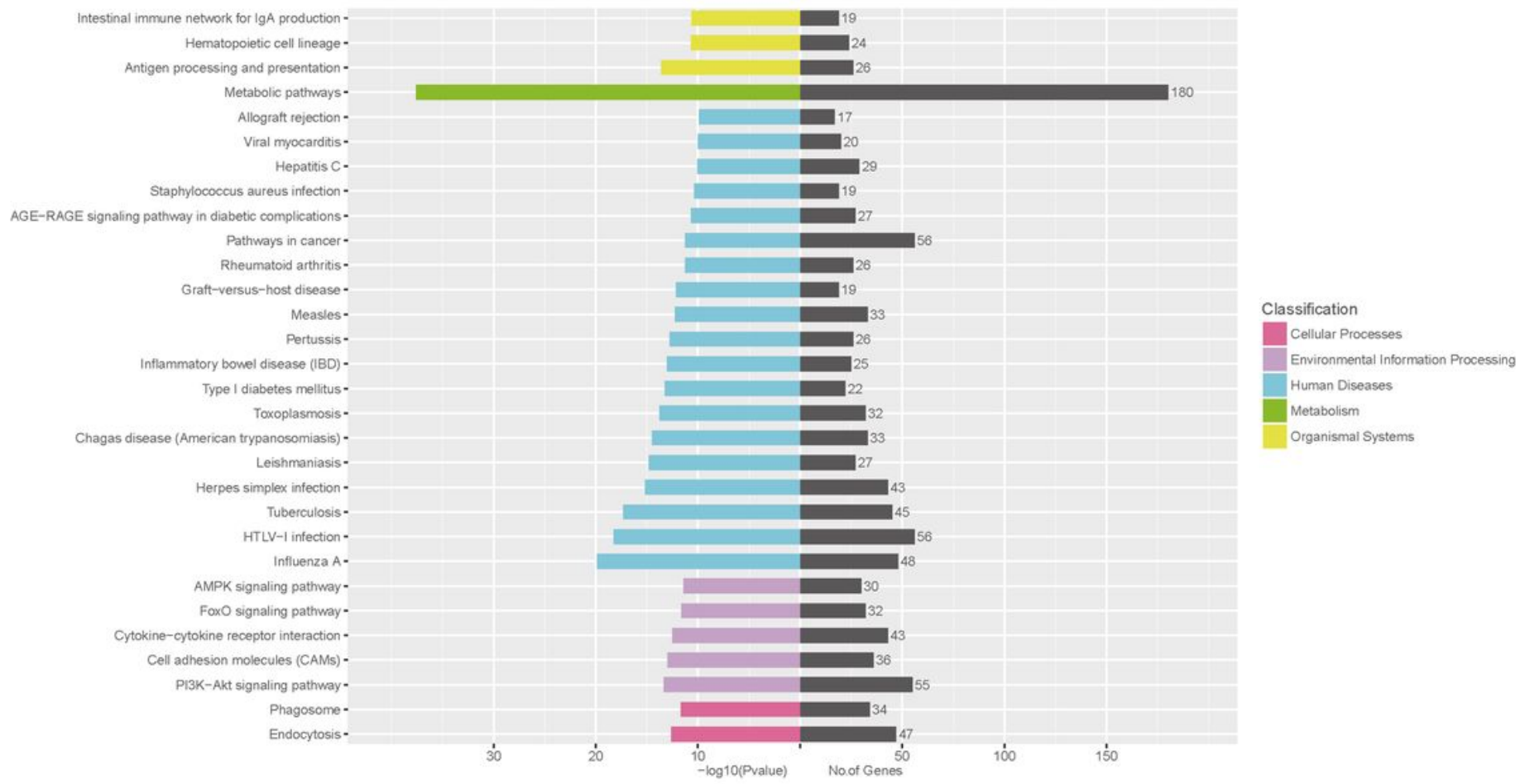

Figure 5

Kyoto Encyclopedia of Genes and Genomes pathways. KEGG pathway enrichment analysis of target genes of candidate circRNAs. 


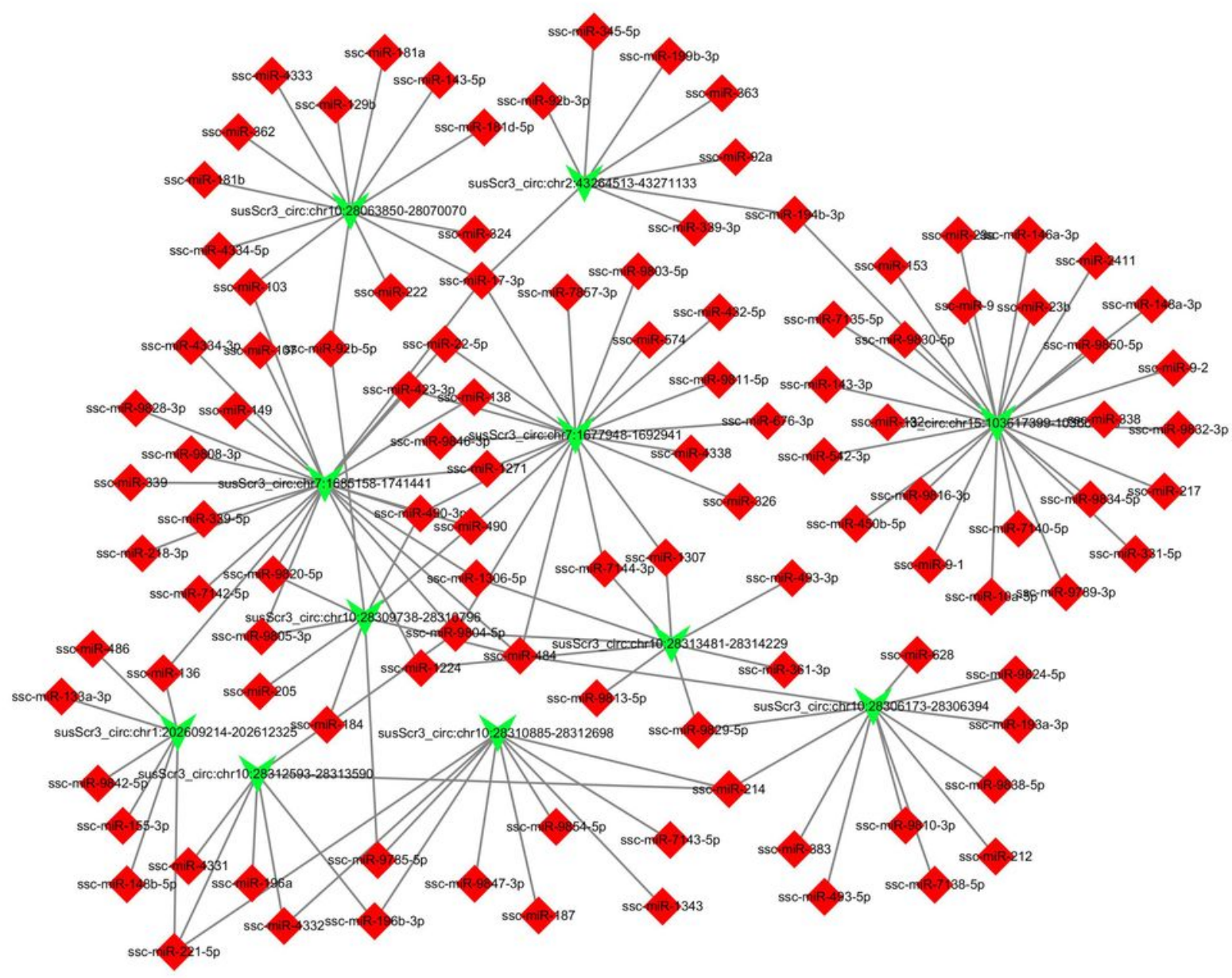

Figure 5

Co-expression network of the miRNAs and candidate circRNAs. Red squares represent miRNAs. Green arrows represent circRNAs. 


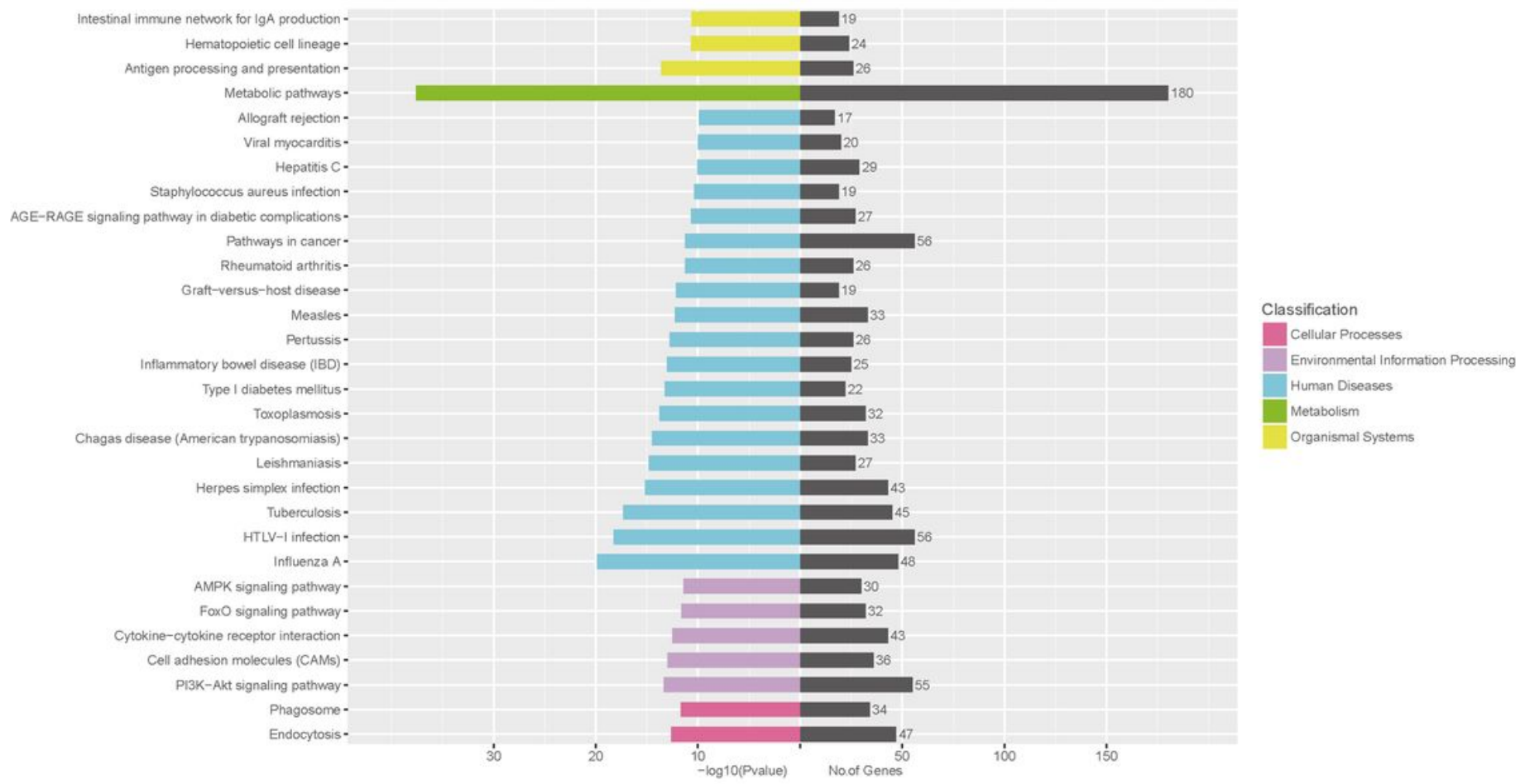

Figure 6

Kyoto Encyclopedia of Genes and Genomes pathways. KEGG pathway enrichment analysis of target genes of candidate circRNAs. 
circBRWD1

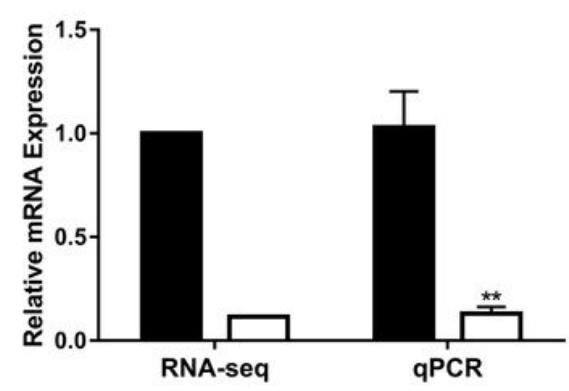

circIGFN1a

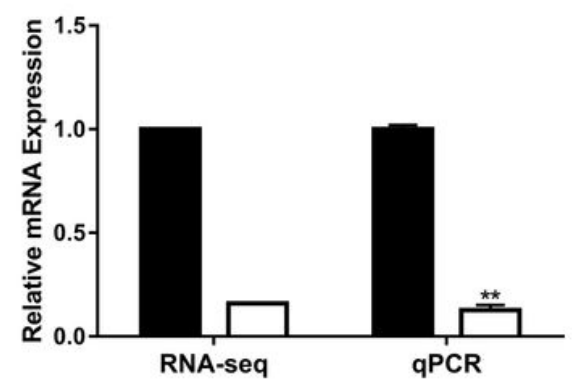

circIGFN1d

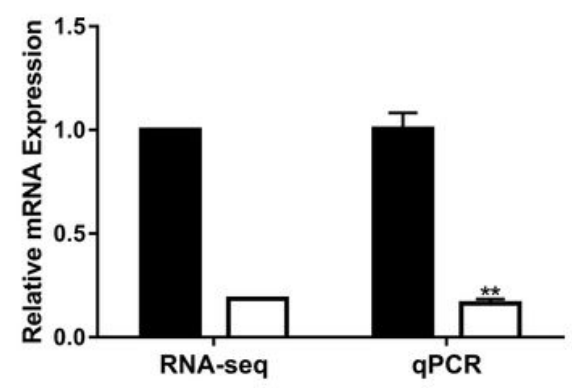

circCSRP3

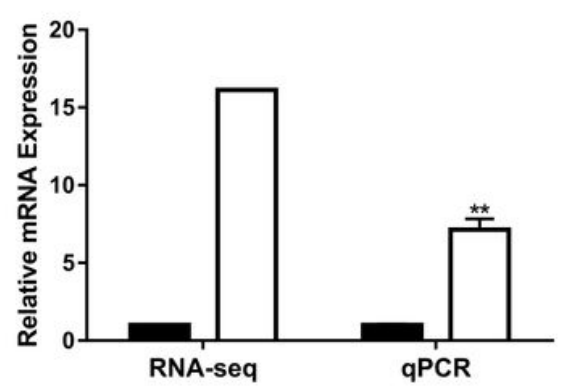

circIGFN1b

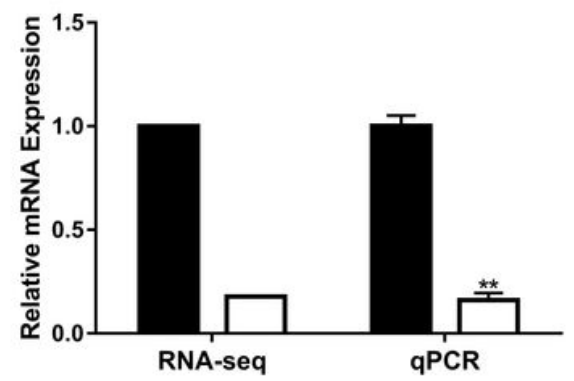

circIGFN1e

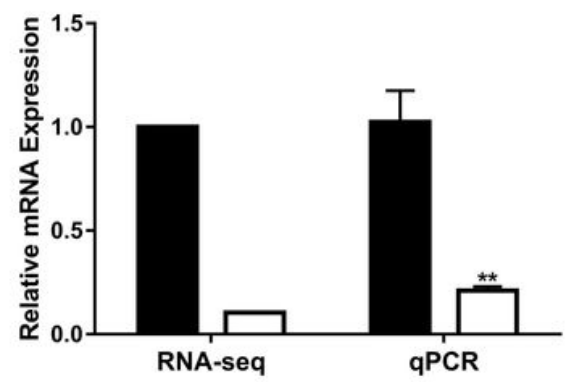

circMYLK4

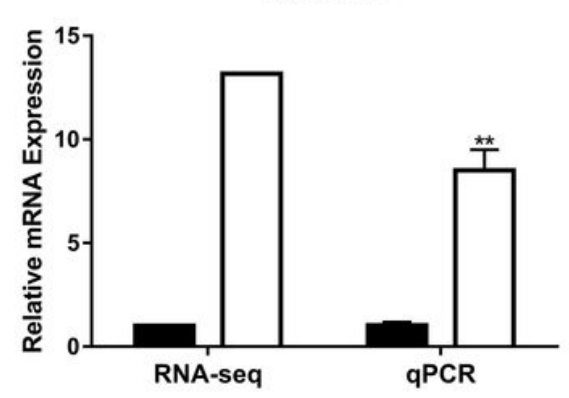

circIGFN1c

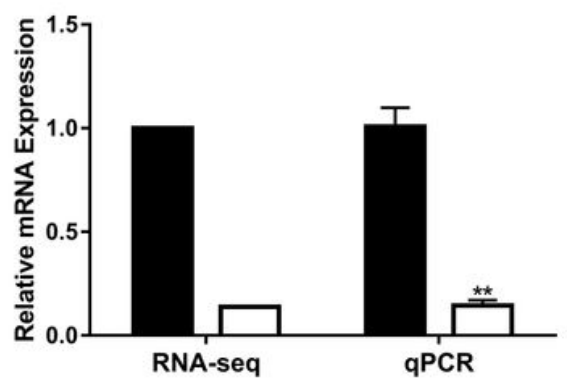

circIGFN1f

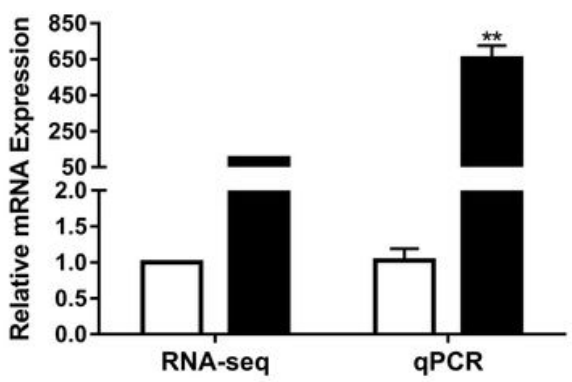

Figure 6

RT-qPCR validation of nine differentially expressed circular RNAs between LD and Sol. Black indicates the tissue of LD, and white indicates the Sol. The data presented in the $Y$ axis represent the relative expression of both RPM and RT-qPCR and are expressed as the mean $\pm S E M$. ${ }^{*} P<0.05, * * P<0.01 . N=3$. 


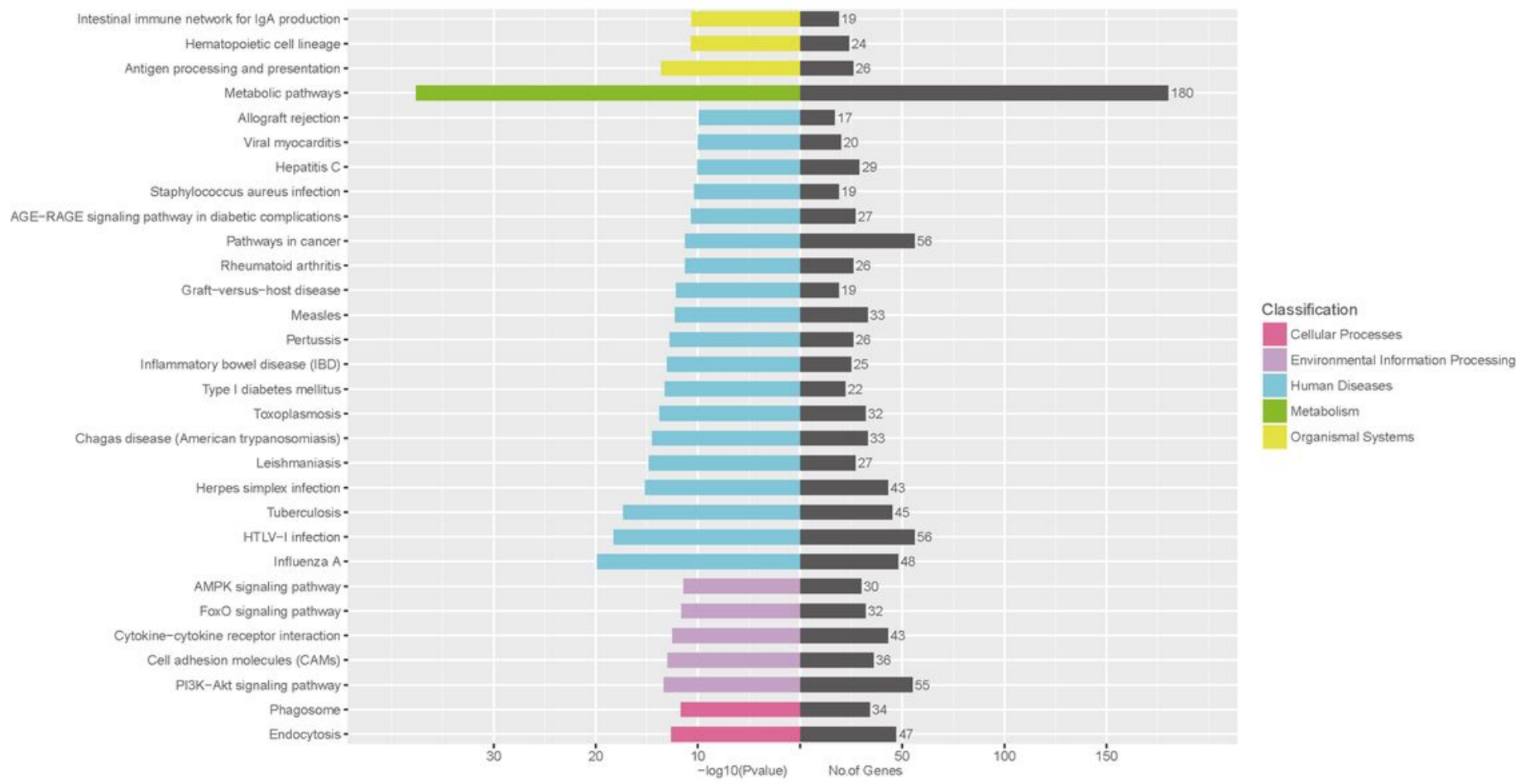

Figure 6

Kyoto Encyclopedia of Genes and Genomes pathways. KEGG pathway enrichment analysis of target genes of candidate circRNAs. 
circBRWD1

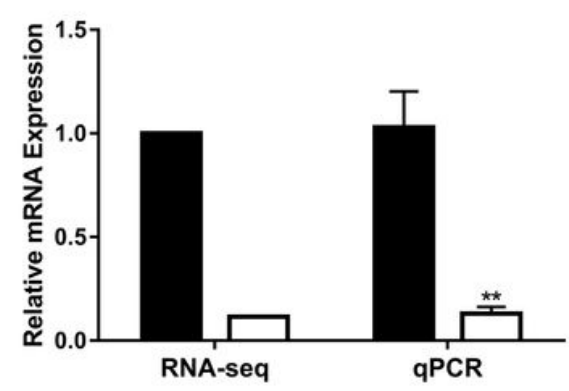

circIGFN1a

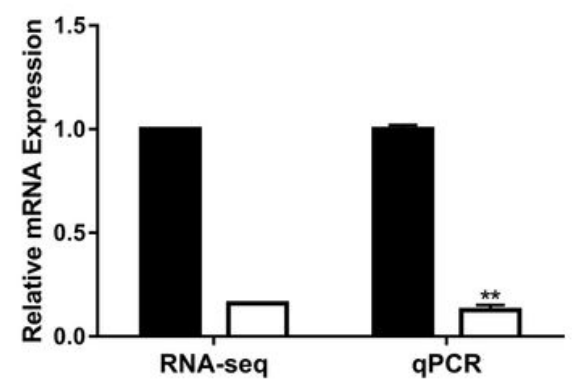

circIGFN1d

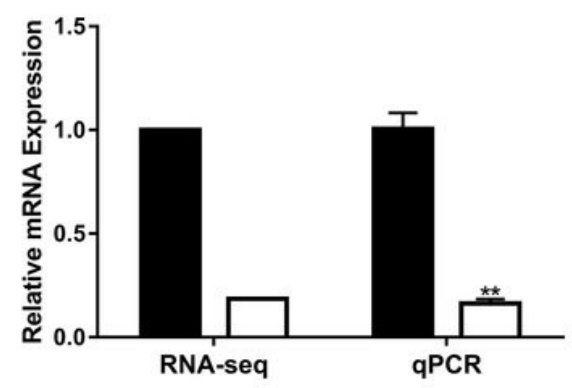

circCSRP3

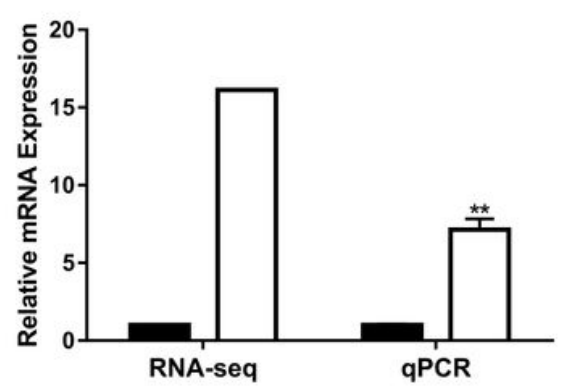

circIGFN1b

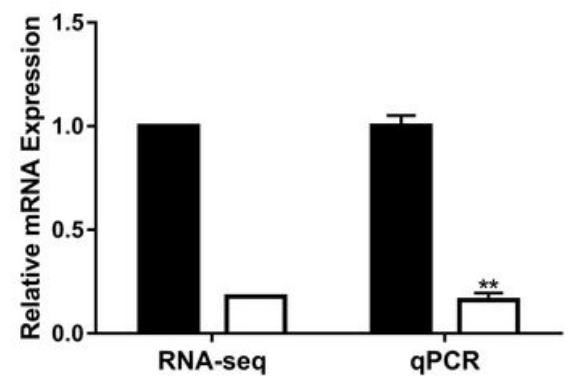

circIGFN1e

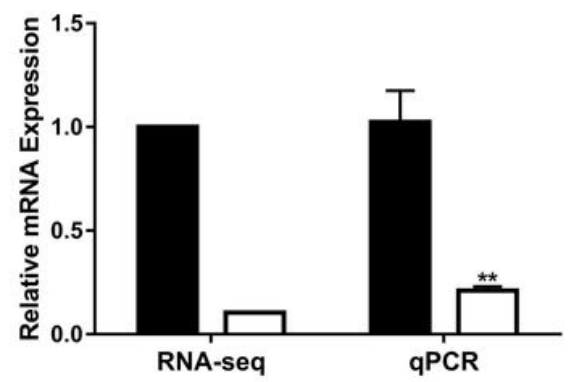

circMYLK4

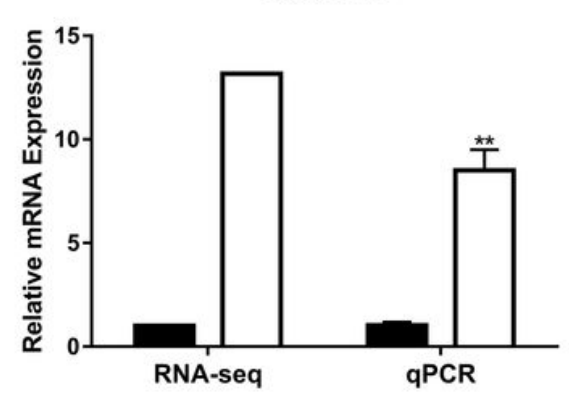

circIGFN1c

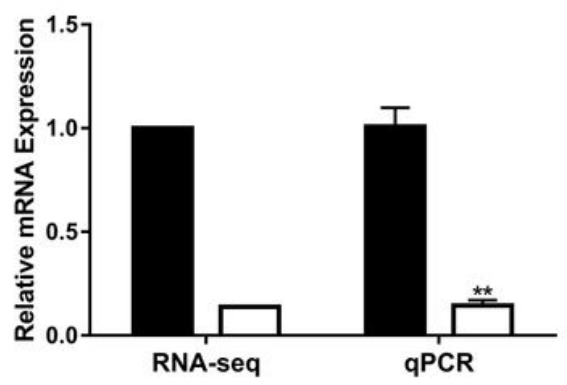

circIGFN1f

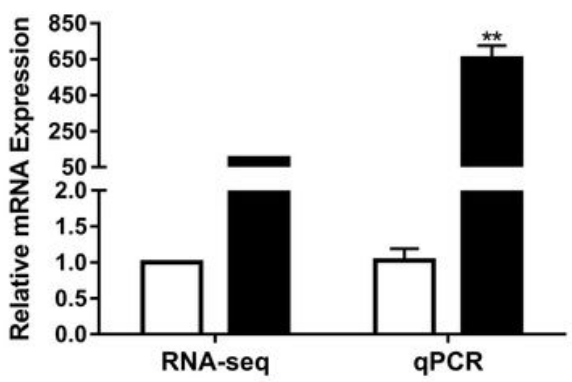

Figure 7

RT-qPCR validation of nine differentially expressed circular RNAs between LD and Sol. Black indicates the tissue of LD, and white indicates the Sol. The data presented in the $Y$ axis represent the relative expression of both RPM and RT-qPCR and are expressed as the mean \pm SEM. ${ }^{*} P<0.05,{ }^{\star} * P<0.01 . N=3$. 
A

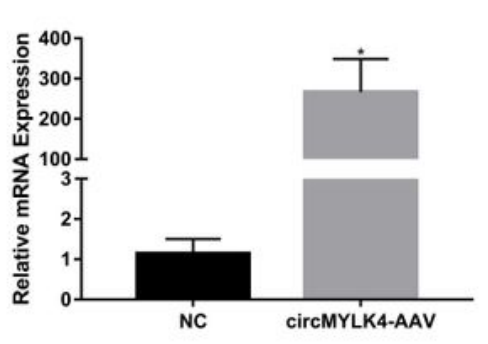

$\mathrm{B}$

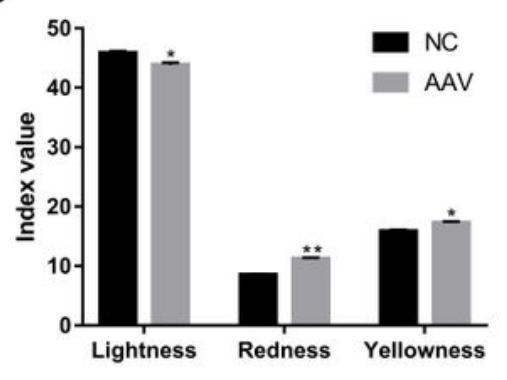

C

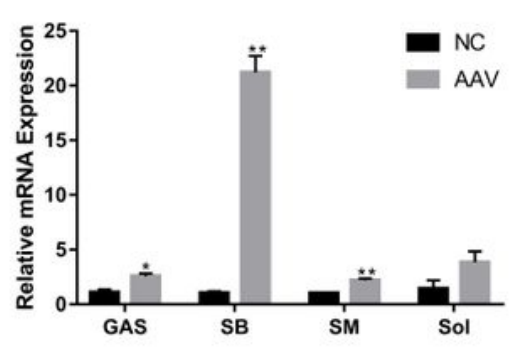

D
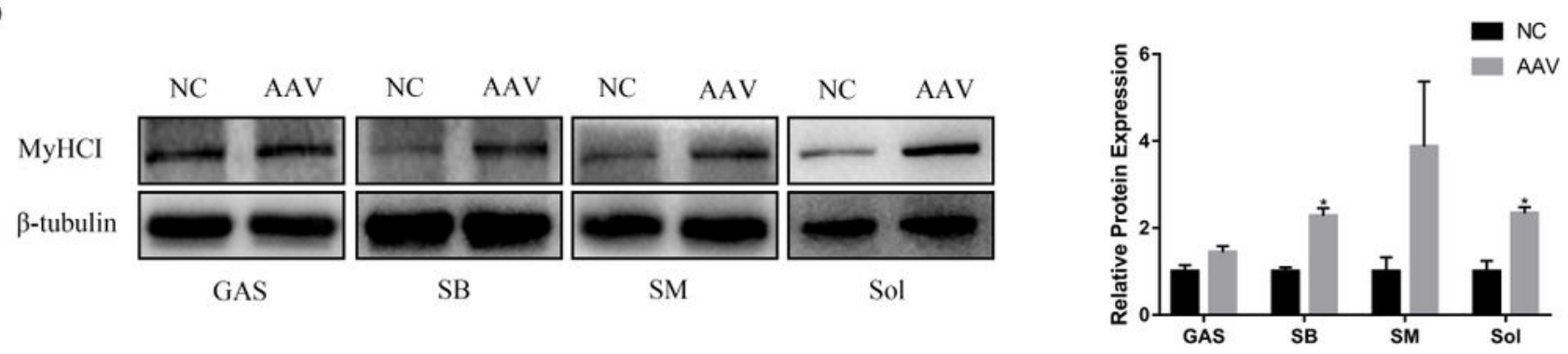

E

GAS

F

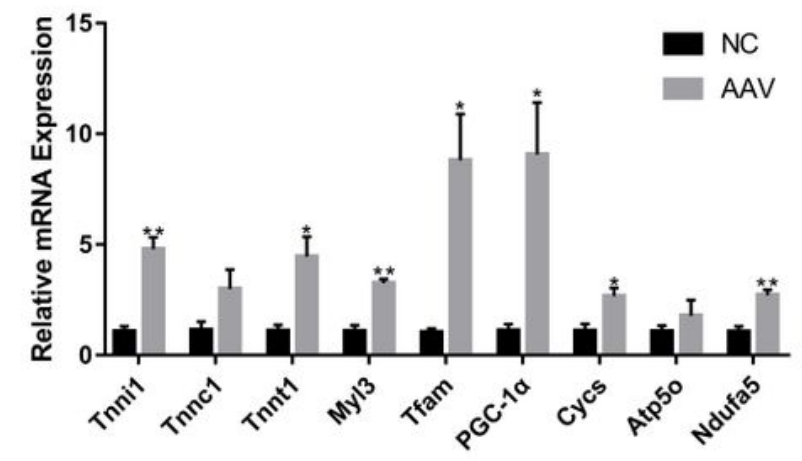

G

SM

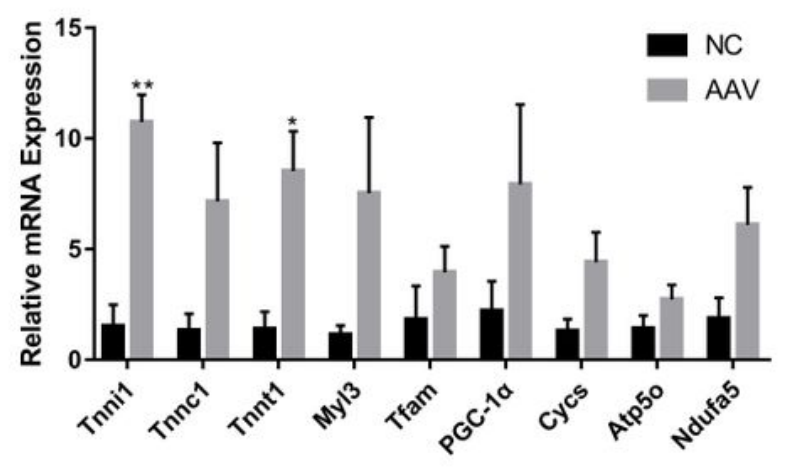

H

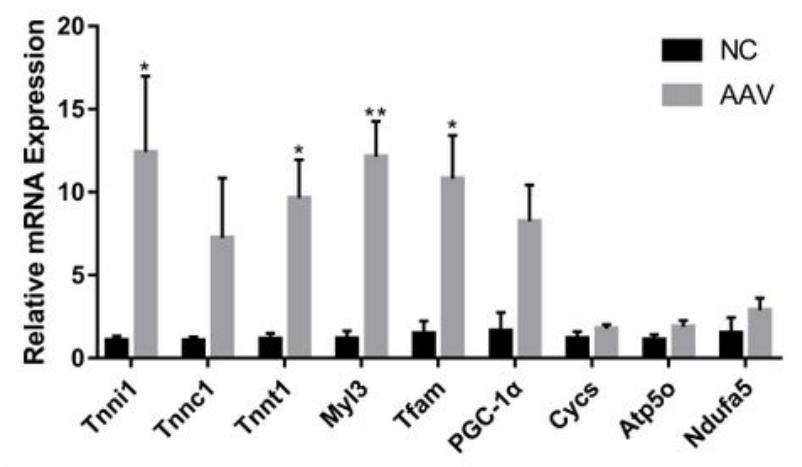

Sol

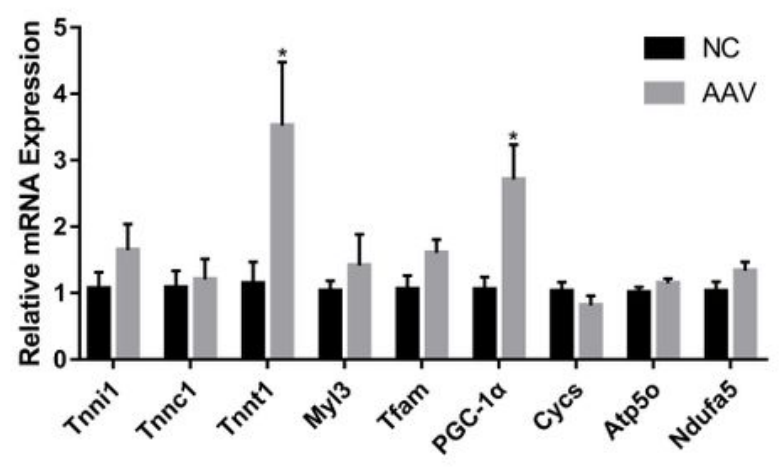

Figure 7

circMYLK4 promotes the formation of slow muscle fibers. (A) Relative expression levels of circMYLK4 in AAV and control groups. (B) Skeletal muscle color test results after overexpression of circMYLK4. (C) The mRNA expression levels of MyHC I in different skeletal muscle tissues after overexpression of circMYLK4. (D) The protein expression and gradation analysis results of $\mathrm{MyHC} \mathrm{I} \mathrm{in} \mathrm{different} \mathrm{skeletal} \mathrm{muscle} \mathrm{tissues}$ after overexpression of circMYLK4. Full-length blots/gels are presented in Supplementary Fig. S1. (E-H) 
The mRNA expression of slow fiber-related genes and mitochondria-related genes in different skeletal muscle tissues after overexpression of circMYLK4.

circBRWD1

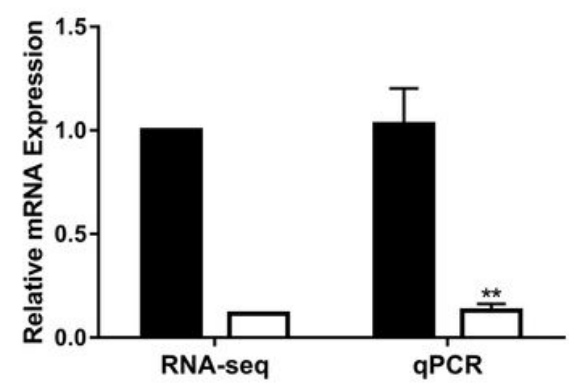

circIGFN1a

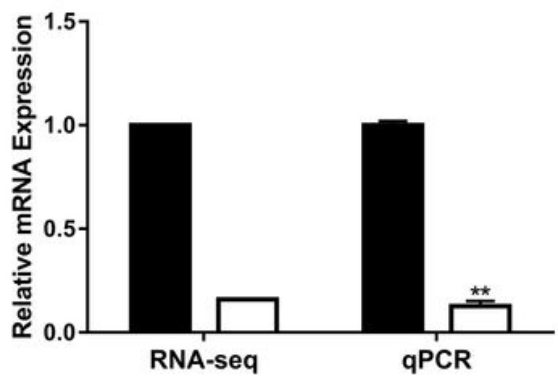

circIGFN1d

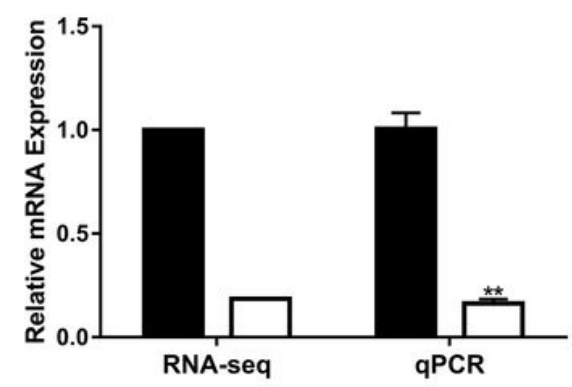

circCSRP3

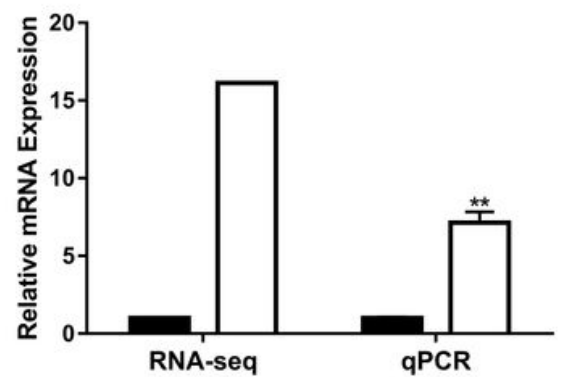

circIGFN1b

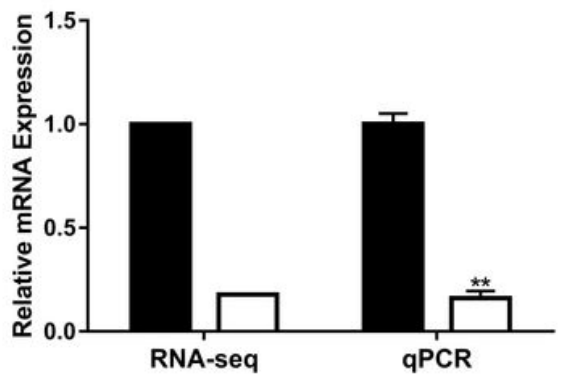

circIGFN1e

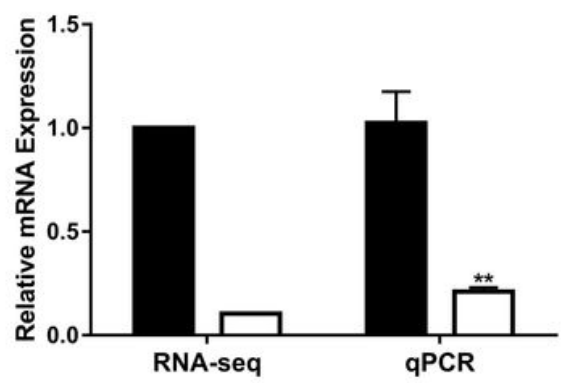

circMYLK4

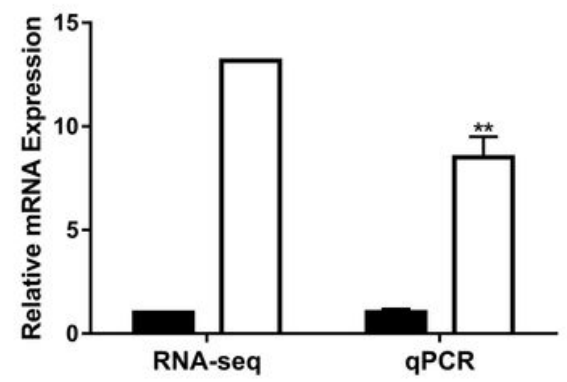

circIGFN1c

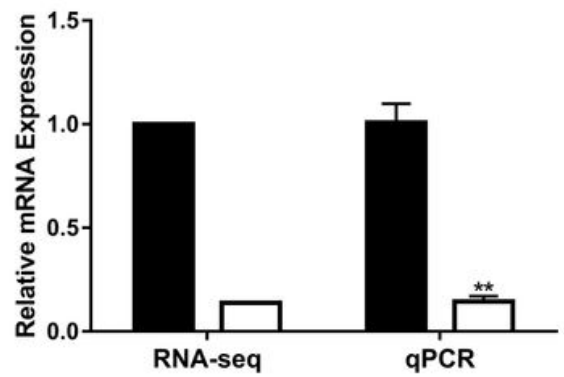

circIGFN1f

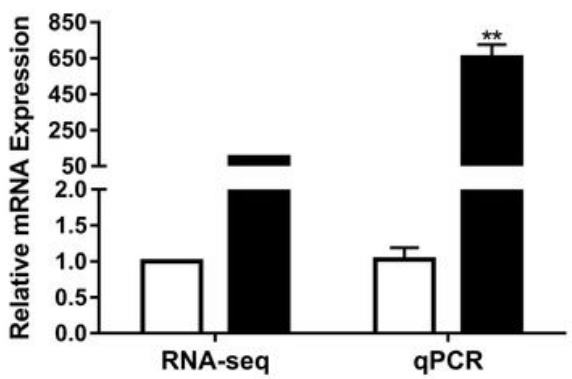

Figure 7

RT-qPCR validation of nine differentially expressed circular RNAs between LD and Sol. Black indicates the tissue of LD, and white indicates the Sol. The data presented in the $Y$ axis represent the relative expression of both RPM and RT-qPCR and are expressed as the mean \pm SEM. ${ }^{*} P<0.05,{ }^{*} P<0.01 . N=3$. 
A

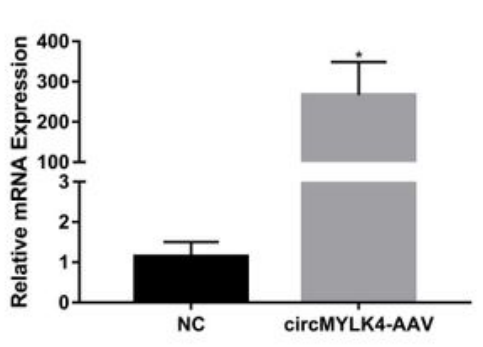

$\mathrm{B}$

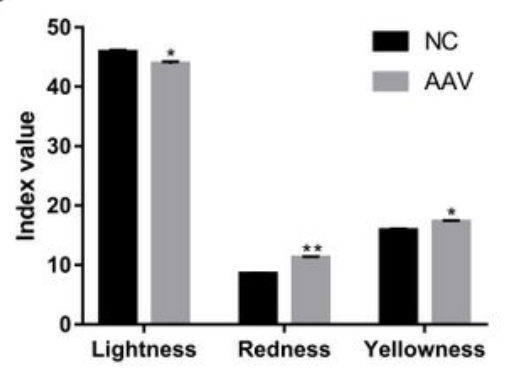

C

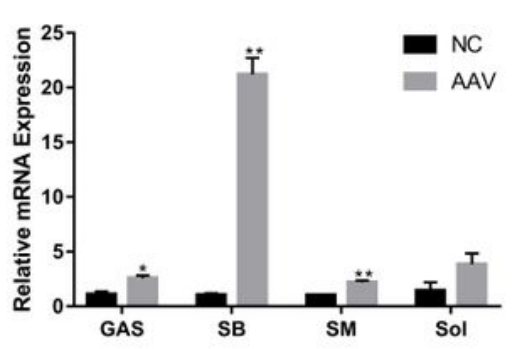

D
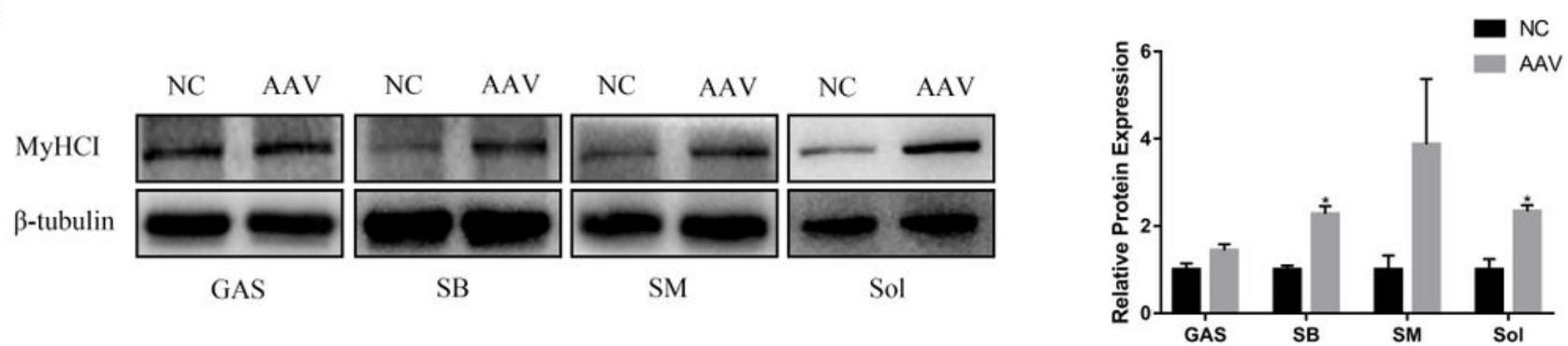

E

GAS

F

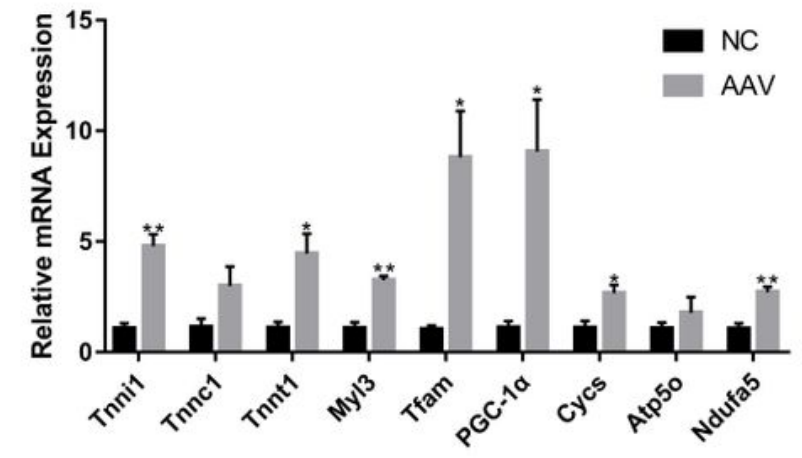

G

SM

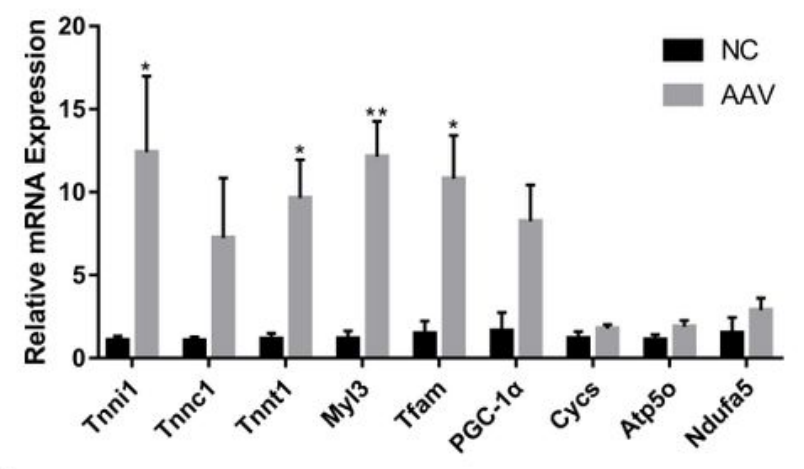

H
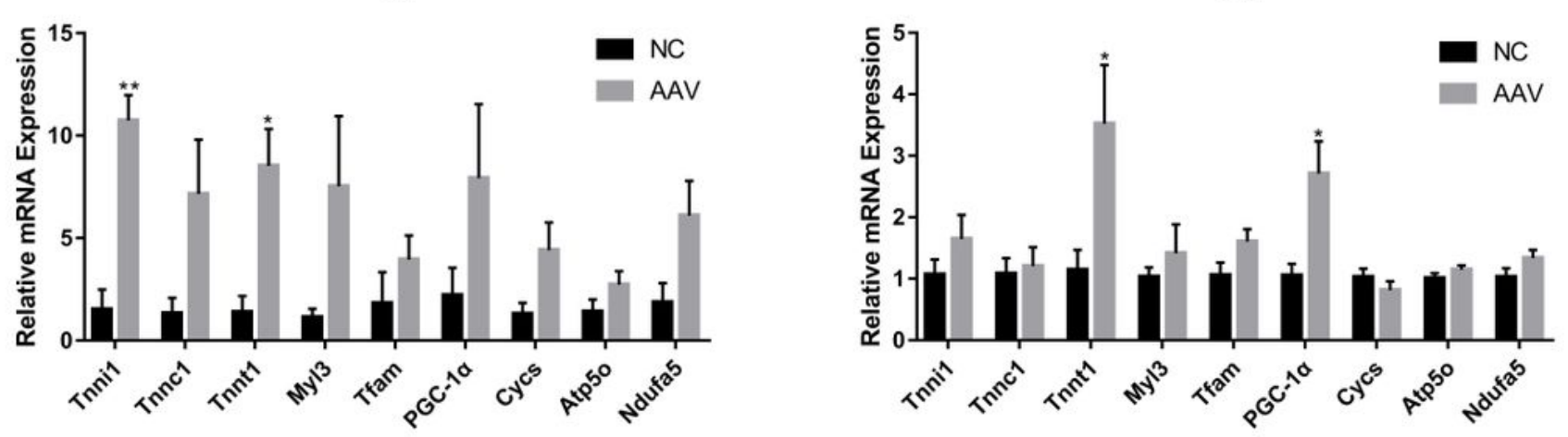

Figure 7

circMYLK4 promotes the formation of slow muscle fibers. (A) Relative expression levels of circMYLK4 in AAV and control groups. (B) Skeletal muscle color test results after overexpression of circMYLK4. (C) The mRNA expression levels of MyHC I in different skeletal muscle tissues after overexpression of circMYLK4. (D) The protein expression and gradation analysis results of $\mathrm{MyHC} \mathrm{I} \mathrm{in} \mathrm{different} \mathrm{skeletal} \mathrm{muscle} \mathrm{tissues}$ after overexpression of circMYLK4. Full-length blots/gels are presented in Supplementary Fig. S1. (E-H) 
The mRNA expression of slow fiber-related genes and mitochondria-related genes in different skeletal muscle tissues after overexpression of circMYLK4.
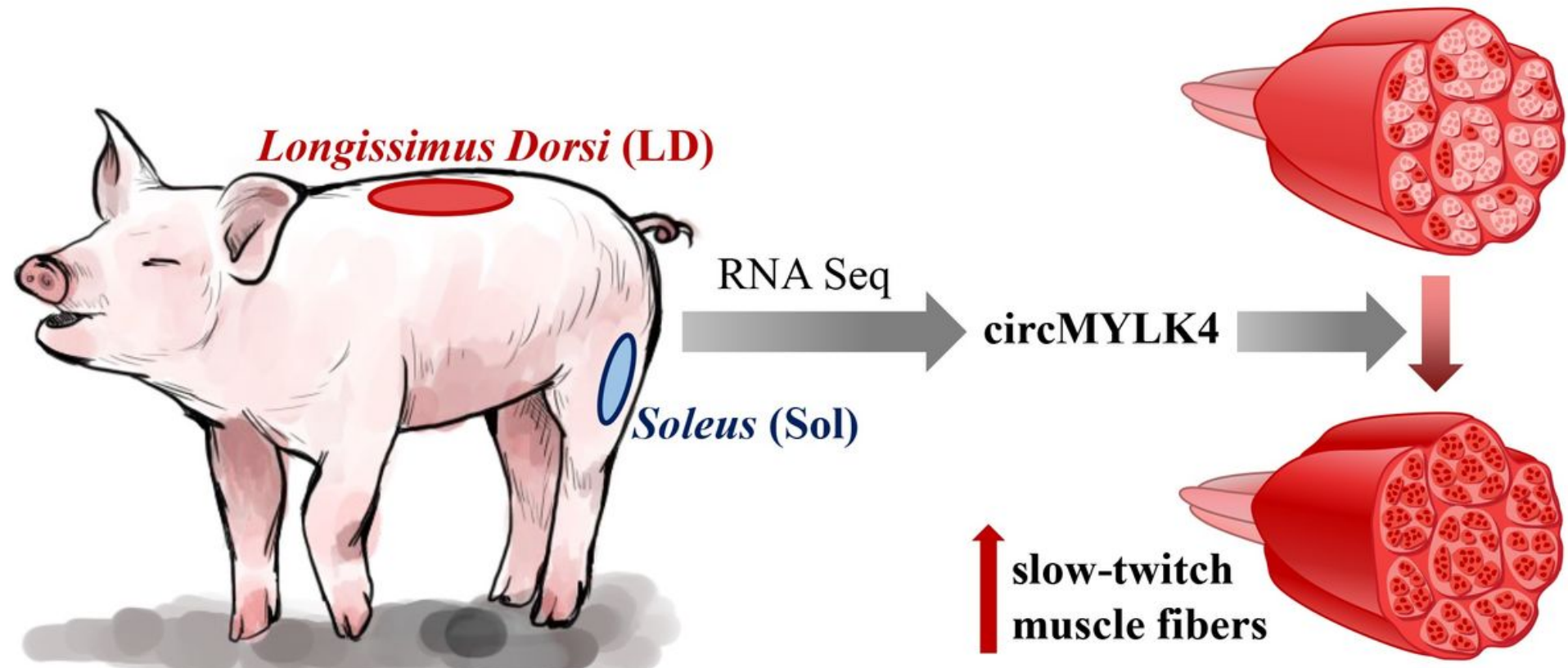

slow-twitch muscle fibers

Figure 9

Schematic diagram of circMYLK4 affecting muscle fiber types

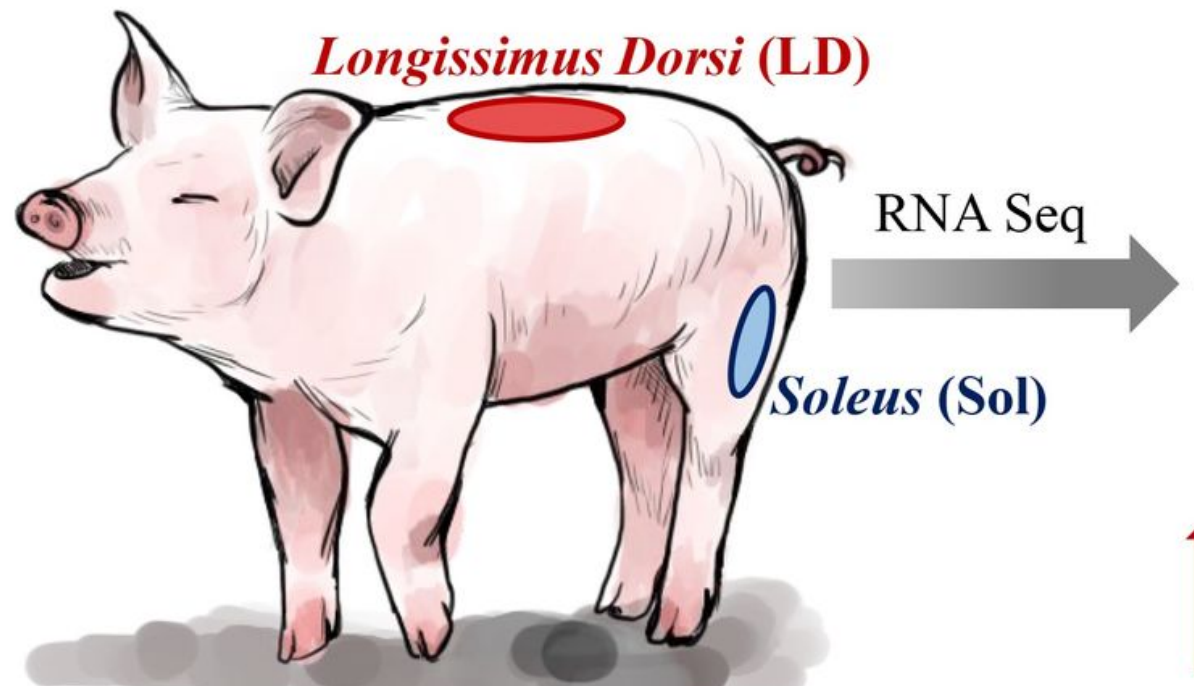
circMYLK4

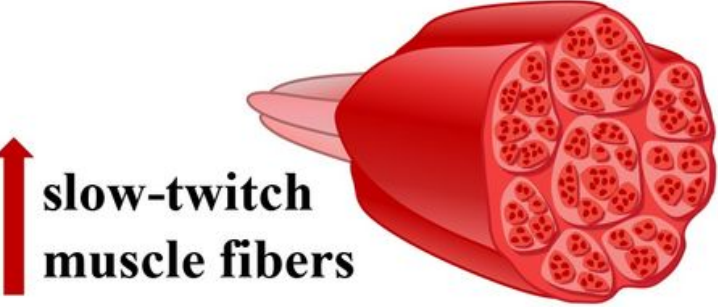

Figure 9

Schematic diagram of circMYLK4 affecting muscle fiber types muscle fibers

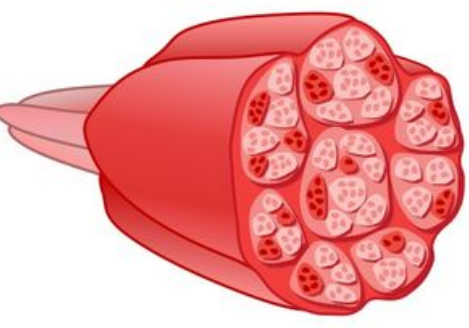

\section{$\operatorname{circMYLK4}$}

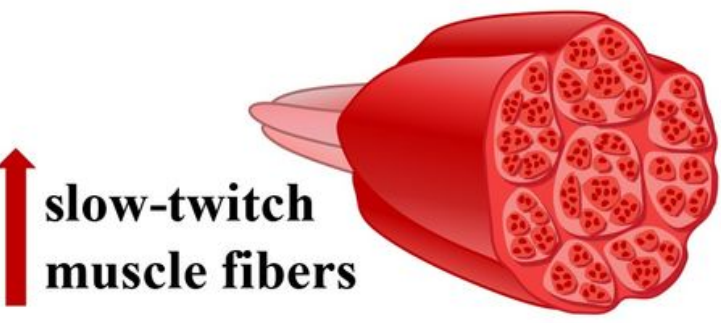



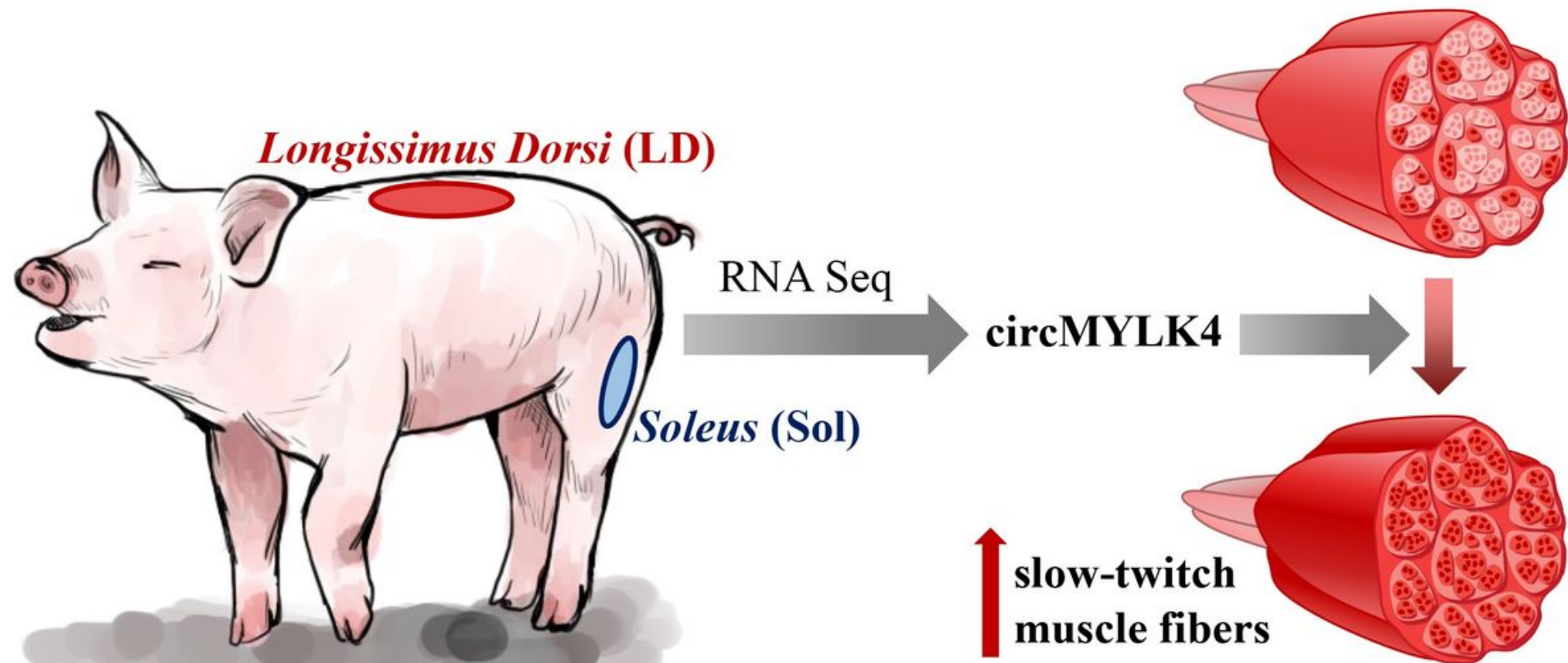

\section{circMYLK4}

slow-twitch

muscle fibers

Figure 9

Schematic diagram of circMYLK4 affecting muscle fiber types

\section{Supplementary Files}

This is a list of supplementary files associated with this preprint. Click to download.

- Additionalfiles.xlsx

- Additionalfiles.xlsx

- Additionalfiles.xlsx

- Supplementaryinformation.doc

- Supplementaryinformation.doc 